\title{
journal
}

\section{Magnetic-Field-Induced Ferroelectric Switching in Multiferroic Aurivillius Phase Thin Films at Room Temperature}

\begin{tabular}{|r|l|}
\hline Journal: & Journal of the American Ceramic Society \\
\hline Manuscript ID: & JACERS-32991.R1 \\
\hline Manuscript Type: & Feature Article \\
\hline Date Submitted by the Author: & n/a \\
\hline Complete List of Authors: & $\begin{array}{l}\text { Keeney, Lynette; Tyndall National Institute, University College Cork } \\
\text { Maity, Tuhin; Tyndall National Institute, University College Cork } \\
\text { Schmidt, Michael; Tyndall National Institute, University College Cork } \\
\text { Amann, Andreas; Tyndall National Institute, University College Cork; } \\
\text { Univeristy College Cork, School of Mathematic Sciences } \\
\text { Deepak, Nitin; Tyndall National Institute, University College Cork } \\
\text { Petkov, Nikolay; Tyndall National Institute, University College Cork } \\
\text { Roy, Saibal; Tyndall National Institute, University College Cork } \\
\text { Pemble, Martyn; Tyndall National Institute, University College Cork; } \\
\text { Tyndall Natinal Institute, Advanced Materials and Surfaces } \\
\text { Whatmore, Roger; Tyndall National Institute, University College Cork }\end{array}$ \\
\hline Keywords: & $\begin{array}{l}\text { ferroelectricity/ferroelectric materials, piezoelectric materials/properties, } \\
\text { films, multiferroics, magnetoelectrics }\end{array}$ \\
\hline \multicolumn{2}{|c}{} \\
\hline \multicolumn{2}{|c}{} \\
\hline
\end{tabular}




\title{
Magnetic-Field-Induced Ferroelectric Switching in Multiferroic Aurivillius Phase Thin Films at Room Temperature
}

\author{
By Lynette Keeney ${ }^{1 *}$, Tuhin Maity ${ }^{1}$, Michael Schmidt $^{1}$, Andreas Amann ${ }^{1,2}$, Nitin Deepak ${ }^{1}$, \\ Nikolay Petkov ${ }^{1}$, Saibal Roy ${ }^{1}$, Martyn E. Pemble ${ }^{1}$ and Roger W. Whatmore ${ }^{1}$
}

[*] Dr. L. Keeney

E-mail: 1ynette.keeney@,tyndall.ie

Telephone: +353212346391

1. Tyndall National Institute, University College Cork, 'Lee Maltings', Dyke Parade, Cork, Ireland.

2. School of Mathematical Sciences, University College Cork, Ireland.

\begin{abstract}
Keywords: Multiferroic, Magnetoelectric, Ferroelectric, Aurivillius, Piezoresponse force microscopy
\end{abstract}


Single-phase multiferroic materials are of considerable interest for future memory and sensing applications. Thin films of Aurivillius phase $\mathrm{Bi}_{7} \mathrm{Ti}_{3} \mathrm{Fe}_{3} \mathrm{O}_{21}$ and $\mathrm{Bi}_{6} \mathrm{Ti}_{2.8} \mathrm{Fe}_{1.52} \mathrm{Mn}_{0.68} \mathrm{O}_{18}$ (possessing 6 and 5 perovskite units per half-cell respectively) have been prepared by chemical solution deposition on $c$-plane sapphire. Superconducting quantum interference device magnetometry reveal $\mathrm{Bi}_{7} \mathrm{Ti}_{3} \mathrm{Fe}_{3} \mathrm{O}_{21}$ to be antiferromagnetic $\left(\mathrm{T}_{\mathrm{N}}\right.$ $190 \mathrm{~K}$ ) and weakly ferromagnetic below $35 \mathrm{~K}$, however $\mathrm{Bi}_{6} \mathrm{Ti}_{2.8} \mathrm{Fe}_{1.52} \mathrm{Mn}_{0.68} \mathrm{O}_{18}$ gives a distinct room temperature in-plane ferromagnetic signature $\left(\mathrm{M}_{\mathrm{s}}=0.74 \mathrm{emu} / \mathrm{g}, \mu_{0} \mathrm{H}_{\mathrm{c}}=7 \mathrm{mT}\right)$. Microstructural analysis, coupled with the use of a statistical analysis of the data, allows us to conclude that ferromagnetism does not originate from second phase inclusions, with a confidence level of 99.5\%. Piezoresponse force microscopy (PFM) demonstrates room temperature ferroelectricity in both films, whereas PFM observations on $\mathrm{Bi}_{6} \mathrm{Ti}_{2.8} \mathrm{Fe}_{1.52} \mathrm{Mn}_{0.68} \mathrm{O}_{18}$ show Aurivillius grains undergo ferroelectric domain polarization switching induced by an applied magnetic field. Here we show for the first time that $\mathrm{Bi}_{6} \mathrm{Ti}_{2.8} \mathrm{Fe}_{1.52} \mathrm{Mn}_{0.68} \mathrm{O}_{18}$ thin films are both ferroelectric and ferromagnetic and, demonstrate magnetic field induced switching of ferroelectric polarization in individual Aurivillius phase grains at room temperature. 


\section{Introduction}

As the use of computers continues to expand rapidly there is increasingly a need for data storage technologies with higher densities, non-volatility and lower power consumption. ${ }^{1}$ Single-phase, room temperature magnetoelectric multiferroic materials are of considerable interest for such applications $\mathrm{s}^{2,3,4,5,6,7,8}$. However, materials that are magnetoelectric at room temperature are very unusual ${ }^{9}$ (see Sidebar A.). The perovskite ferroelectric $\mathrm{BiFeO}_{3}$ exhibits antiferromagnetic ordering at ambient temperature ${ }^{10}$ and its electric polarization has been used to control antiferromagnetic ordering ${ }^{11,12,13}$, but there is no evidence as of yet that its ferroelectric polarization can be switched by a magnetic field. There has, therefore, been an intense search for room temperature magnetoelectric multiferroics within which the coupling of ferroelectric and ferromagnetic polarisations might be demonstrated. 


\section{$\underline{\text { Sidebar A. Multiferroics and Magnetoelectrics }}$}

Ferroelectric materials form a subset of the polar electrically polarizable materials for which the electrical dipole moments within their structure can be switched between at least two stable states (e.g. up and down) by an external electric field. Ferromagnets form a subset of the magnetically polarizable materials (Sidebar D.). Materials which demonstrate both ferroelectric and ferromagnetic properties within the same phase are known as multiferroic materials. $^{14,15,16,17,18,19}$ Magnetoelectric coupling refers to the induction of magnetization by an electric field, or vice versa, and may arise through direct coupling between magnetic and electric polarizations in a single material, or indirectly via strain-mediated coupling in a multi-phase material. ${ }^{3,20,21,22,23,24,25}$ Such strain-mediated indirect magnetoelectric coupling in composite materials can occur, for example, through a magnetostrictive strain (induced in one phase by a change in applied magnetic field) coupling to a piezoelectrically-induced polarization change in a second phase mechanically-coupled to the first. The SI unit of the magnetoelectric coupling coefficient, $\alpha$, is $\left[\mathrm{sm}^{-1}\right]$ which can be converted to the technical unit $\left[\mathrm{Vcm}^{-1} \mathrm{Oe}^{-1}\right]$ if the permittivity $(\varepsilon)$ of the given material is known: $\left[\mathrm{sm}^{-1}\right]=1.1 \times 10^{-11} \varepsilon\left[\mathrm{Vcm}^{-}\right.$ $\left.{ }^{1} \mathrm{Oe}^{-1}\right]$. A magnetoelectric coupling coefficient of $5.90 \mathrm{Vcm}^{-1} \mathrm{Oe}^{-1}$ has been reported for laminate complexes of lead zirconate titanate $(\mathrm{PZT})$ and Terfenol-D $\left(\mathrm{TbDyFe}_{2}\right)$ by straining the magnetostrictive phase under a DC magnetic bias of $4.2 \mathrm{~T},{ }^{26}$ which induces stress on the piezoelectric phase, generating an electric field in the piezoelectric phase. Magnetic force microscopy imaging of $\left(\mathrm{BiFeO}_{3}\right)_{0.65}-\left(\mathrm{CoFe}_{2} \mathrm{O}_{4}\right)_{0.35}$ nanostructured composite heterostructures ${ }^{27}$, demonstrated two electrically-switchable perpendicular magnetic states at ambient conditions for ferromagnetic $\mathrm{CoFe}_{2} \mathrm{O}_{4}$ nanopillars embedded in $\mathrm{BiFeO}_{3}$. Additionally, the magnetoelectric coupling effect becomes controllable in a weak 


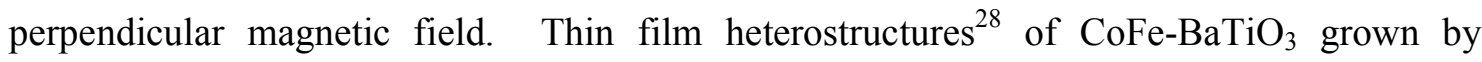
electron-beam evaporation exhibit giant magnetoelectric coupling coefficients $\left(3 \times 10^{-6} \mathrm{sm}^{-1}\right)$ at room temperature. Lahtinen et al. demonstrated that it is possible to precisely write and erase regular ferromagnetic domain patterns and to control the motion of magnetic domain walls in small electric fields over large areas in these composites by strain-mediated correlations between ferromagnetic domain walls and ferroelastic domain boundaries.

Multiferroic coupling in a single phase material can occur when the switching of one order parameter (e.g. ferromagnetic polarization) induces a switching of the other order parameter (in this example ferroelectric polarization). Examples of single-phase magnetoelectrics include: $\mathrm{Cr}_{2} \mathrm{O}_{3}(<260 \mathrm{~K})^{29}, \mathrm{CuO}(<230 \mathrm{~K})^{30}, \mathrm{TbMnO}_{3}(<27 \mathrm{~K})^{31}, \mathrm{Ni}_{3} \mathrm{~B}_{7} \mathrm{O}_{13} \mathrm{I}$ $(<64 \mathrm{~K})^{32}, \mathrm{DyMn}_{2} \mathrm{O}_{5}(<43 \mathrm{~K})^{33}$. The synthesis of novel room temperature single-phase magnetoelectric multiferroic materials is particularly appealing, not only because they have two sets of interesting physical properties with 4 polarization states (positive and negative in both electrical and magnetic polarizations), but also because the multiferroic coupling interactions could lead to a range of potential applications. Such 4-state multiferroic materials could potentially lead to a new generation of rapid, energy efficient magnetoelectric memory devices that can be electrically written and magnetically read, storage of multiple bits per memory element ${ }^{4}$, and magnetic field sensors where the ferromagnetic resonance could be tuned electrically instead of magnetically. ${ }^{21,34}$

However, single-phase magnetoelectric multiferroics are rare, especially at room temperature, due to the competing electronic requirements for ferroelectricity and ferromagnetism ( essential for stabilizing ferroelectric distortion; therefore the cation driving ferroelectricity must formally be in the $d^{0}$ state. Conversely, $d$-orbital occupancy is a requirement for the existence of magnetic ordering. Hill has suggested that the conditions for obtaining 
ferroelectricity and ferromagnetism in a single phase can potentially be met by incorporating $d^{0}$ and $d^{n}$ cations into the same structure.

$\mathrm{Sr}_{3} \mathrm{Co}_{2} \mathrm{~F}_{24} \mathrm{O}_{21}$ demonstrates low-field magnetoelectric effects at room temperature, however shows no polarization at zero magnetic field and therefore is not a bilinear magnetoelectric. ${ }^{35} \mathrm{SrCo}_{2} \mathrm{Ti}_{2} \mathrm{Fe}_{8} \mathrm{O}_{19}$ does exhibit spontaneous polarization at zero magnetic field $\left(\sim 25 \mu \mathrm{Ccm}^{-2}\right)^{5}$ and magnetic force microscopy investigations of this ceramic under various electric fields $\left(\mathrm{E}_{\mathrm{dc}} \sim-20\right.$ to $\left.+20 \mathrm{kv} / \mathrm{cm}\right)$ demonstrated electric-field control of magnetism at room temperature in the absence of a magnetic field bias (converse magnetoelectric effect) and decreases in magnetization of up to $6.3 \%$ on application of a magnetic field of $46 \mathrm{mT}$ and $E_{\mathrm{dc}}$ of $22 \mathrm{kv} / \mathrm{cm}$. Recently, a newly-discovered single-phase multiferroic, $\left[\mathrm{Pb}\left(\mathrm{Zr}_{0.53} \mathrm{Ti}_{0.47}\right) \mathrm{O}_{3}\right]_{0.6}-\left[\mathrm{Pb}\left(\mathrm{Fe}_{0.5} \mathrm{Ta}_{0.5}\right) \mathrm{O}_{3}\right]_{0.4}$ has been shown to exhibit significant ( $\sim 60 \%$ change in polarization) magnetoelectric coupling at room temperature $\left(\sim 1 \times 10^{-7} \mathrm{sm}^{-}\right.$ $\left.{ }^{1}\right)^{36}$, demonstrating that with materials development and design, the development of room temperature multiferroic materials can be achieved. 
The ferroelectric Aurivillius layer-structures ${ }^{37}$, described by general formula $\mathrm{Bi}_{2} \mathrm{O}_{2}\left(A_{\mathrm{m}}\right.$ ${ }_{1} B_{\mathrm{m}} \mathrm{O}_{3 \mathrm{~m}+1}$ ), are naturally 2 -dimensionally nanostructured with large $c$-axis parameters, high Curie temperatures $\left(>600^{\circ} \mathrm{C}\right)$ and large in-plane spontaneous polarisations. The number of $A B \mathrm{O}_{3}$ perovskite units $(\mathrm{m})$ per half-cell can be changed within the range 2 to 9 , depending on composition, and a wide variety of $B$-site cations with +3 to +5 oxidation states accommodated $^{38,39,40,41}$. The system, discussed in greater detail in Sidebar B., offers the potential for including substantial amounts of magnetic cations within a strongly ferroelectric system, and hence the potential for the discovery of new room temperature multiferroics. 


\section{Sidebar B. The Aurivillius family of ferroelectric oxides}

Aurivillius ${ }^{37}$ bismuth-based compounds, sometimes referred to as the layered perovskites and described by the general formula $\mathrm{Bi}_{2} \mathrm{O}_{2}\left(A_{\mathrm{m}-1} B_{\mathrm{m}} \mathrm{O}_{3 \mathrm{~m}+1}\right)$, represent an important class of ferroelectric compounds. The materials are members of an homologous series of Bi-layered oxides, where the structure is a naturally-layered nano-composite. The 2-dimensional nanostructures have large $c$-axis lattice parameters, in the nanometer range, and consist of fluoritestructured $\left(\mathrm{Bi}_{2} \mathrm{O}_{2}\right)^{2+}$ layers of thickness $f$ (typically $\sim 0.4 \mathrm{~nm}$ ) lying in the (001) plane alternating with $\mathrm{mABO}_{3}$ perovskite units in a sandwich type arrangement. The average thickness of the perovskite-type block, $h$, depends on the number of octahedral perovskite units $(m)$ in the block: $h=p m$ where $p$ is the average thickness of the perovskite-like units (also typically $\sim 0.4 \mathrm{~nm}$ ). ${ }^{42}$ (Note that this is only an approximation, as octahedral tilting, and choice of A \& B cations will change the average height of each perovskite unit. ${ }^{43,44,45}$ ) The value of $m$ can be integer or fractional. ${ }^{14}$ Fractional values of $m$ usually occur with "mixtures" between a pure Aurivillius phase compound and a perovskite end member and are formed by recurrent intergrowth of the perovskite blocks of two Aurivillius end-members, eg. $\mathrm{BaBi}_{8} \mathrm{Ti}_{7} \mathrm{O}_{27}(m=3.5)$ is formed from $\left(\mathrm{Bi}_{4} \mathrm{Ti}_{3} \mathrm{O}_{12}\right)_{0.75}-\left(\mathrm{BaTiO}_{3}\right)_{0.25}{ }^{46,47}$ The values of $f$ and $h$ are related to the cell parameter by $f+h=c / 2$ (Fig. B.1.).

The layered-structured Aurivillius phase materials are a particularly attractive class of oxides as their structure allows the design and synthesis of new materials in thin film form with interesting electrical and magnetic properties. Between the bismuth oxide layers, the number of octahedral layers can be increased and a homologous series of compounds with the general formula $\mathrm{Bi}_{\mathrm{m}+1} \mathrm{Fe}_{\mathrm{m}-3} \mathrm{Ti}_{3} \mathrm{O}_{3 \mathrm{~m}+3}(m=4$ to 9$)$ has been realized by inserting bismuth 
ferrite units, $\mathrm{BiFeO}_{3}$, into 3-layered bismuth titanate, $\mathrm{Bi}_{4} \mathrm{Ti}_{3} \mathrm{O}_{12}$. In Fig. B.1.(a) 3 units of $\mathrm{BiFeO}_{3}$ have been inserted into $\mathrm{Bi}_{4} \mathrm{Ti}_{3} \mathrm{O}_{12}$ to form the 6-layered material, $\mathrm{Bi}_{7} \mathrm{Ti}_{3} \mathrm{FeO}_{21}$.

For this homologous series, Lomanova et al. ${ }^{42}$ have pointed out that, as the number of perovskite-like layers increases, the cell $c$ parameter rises almost linearly, implying that the perovskite-like units incorporated into the Aurivillius phase structure experience only slight changes along the $c$ axis with increasing $m$. For this series, an average thicknesses of the perovskite layers, $p \cong 4.11 \AA$ and the fluorite layers, $f \cong 4.08 \AA$ was estimated. ${ }^{42}$

The Fe distribution over the two non-equivalent octahedral $B$ cation sites in the perovskite block (identified as $\mathrm{B}(1)$ inside the block and $\mathrm{B}(2)$ for the octahedra on the outer sides of the block adjacent to the $\mathrm{Bi}_{2} \mathrm{O}_{2}{ }^{2-}$ layers) has been investigated for the $\mathrm{Bi}_{\mathrm{m}+1} \mathrm{Fe}_{\mathrm{m}}$ ${ }_{3} \mathrm{Ti}_{3} \mathrm{O}_{3 \mathrm{~m}+3}$ series. ${ }^{14}$ For $m=3.5$ to $7, \mathrm{Fe}^{3+}$ ions preferentially occupy the $\mathrm{B}(1)$ sites, however the ordered distribution of ions over $\mathrm{B}(1)$ and $\mathrm{B}(2)$ sites decreases with the increase in the perovskite-like block thickness. At $\mathrm{m} \geq 7$, the distribution of $\mathrm{Fe}^{3+}$ and $\mathrm{Ti}^{4+}$ ions of the perovskite-type block tends to become more random and when the value of $m$ increases up to $8-9$, concentrations of ions at $\mathrm{B}(1)$ and $\mathrm{B}(2)$ sites equalize. ${ }^{14}$

On increasing the number of perovskite layers $(m)$, the microstructural, magnetic and physical properties of the materials can be altered significantly. ${ }^{38}$ The layered nature of these materials also allows for the incorporation of significant amounts of magnetic ions with +3 to +5 oxidation states ${ }^{48}$ within the $m A B \mathrm{O}_{3}$ perovskite units. In this way, the normallyconflicting electronic structure requirements for ferroelectricity (unoccupied $d$ orbitals, $d^{0}$ ) and ferromagnetism (partially filled $d$ orbitals, $d^{\mathrm{n}}$ ) in a single phase ${ }^{9}$ can potentially be circumvented and the fabrication of single-phase magnetoelectric multiferroic materials could conceivably be accommodated. 
Lomanova et $\mathrm{al}^{42}$ explored ceramics with general formula $\mathrm{Bi}_{\mathrm{m}-1} \mathrm{Fe}_{\mathrm{m}-3} \mathrm{Ti}_{3} \mathrm{O}_{3 \mathrm{~m}+1}$, and demonstrated the existence of structures with $m$ from 4 to 9 , including some with fractional $m$. Weak room temperature ferromagnetism has been reported for $m=4^{49}$, and antiferromagnetism ( 80 to $300 \mathrm{~K}$ ) for $m=6^{50}$ and $m=7^{51}$. Zurbuchen et $\mathrm{al}^{52}$ showed that the manganese analogue of this system with $m=6$ was ferromagnetic below $55 \mathrm{~K}$, but not ferroelectric. Ferroelectricity and ferromagnetism above room temperature was reported for cobalt-substituted, 4-layered $\mathrm{Bi}_{5} \mathrm{Ti}_{3} \mathrm{Fe}_{0.5} \mathrm{Co}_{0.5} \mathrm{O}_{15}$ ceramic ${ }^{53}$, with a small remanent magnetisation. Subsequent investigations ${ }^{23,34,54,55}$ of $\mathrm{Bi}_{5} \mathrm{Ti}_{3} \mathrm{Fe}_{0.5} \mathrm{Co}_{0.5} \mathrm{O}_{15}$ ceramics and $\mathrm{Bi}_{5} \mathrm{Ti}_{3} \mathrm{Fe}_{0.7} \mathrm{Co}_{0.3} \mathrm{O}_{15}$ films also demonstrated ferroelectric and ferromagnetic behavior at room temperature. However, detailed phase analyses detected trace levels of $\mathrm{CoFe}_{2-\mathrm{x}} \mathrm{Ti}_{\mathrm{x}} \mathrm{O}_{4}$ second phase inclusions, not observed by XRD, but which accounted for the observed magnetization. Indeed, a remanent magnetisation of $7.8 \mathrm{memu} / \mathrm{g}$ as observed by Mao et al. ${ }^{53}$ would correspond to a trace $\mathrm{CoFe}_{2} \mathrm{O}_{4}$ second (or impurity) phase level of only 0.03 wt.\%, which would be very hard to see by any microanalytical method. This observation clearly demonstrates the difficulty of unambiguous assignment of magnetic effects to the parent Aurivillius phase. Other work ${ }^{56}$ has reported ferroelectric and ferromagnetic behavior in thin films of $\mathrm{Bi}_{4.15} \mathrm{Nd}_{0.85} \mathrm{Ti}_{3} \mathrm{Fe}_{0.5} \mathrm{Co}_{0.5} \mathrm{O}_{15}$. Compounds with higher values of $m$, such as $\mathrm{Bi}_{6} \mathrm{Ti}_{3} \mathrm{Fe}_{2} \mathrm{O}_{18}(\mathrm{~B} 6 \mathrm{TFO}, m=5)$, provide a means for increasing the proportion of magnetic cations. Weak ferromagnetic/antiferromagnetic behavior was reported in rare-earth and Co doped $\mathrm{B} \mathrm{TFO} \mathrm{O}^{57,58,59,60}$ ceramics and thin films, but none of the work presented phase analyses at a level which would exclude the possibility that the ferromagnetic responses were due to trace-level second phases.

In this study, thin films of $\mathrm{Bi}_{7} \mathrm{Ti}_{3} \mathrm{Fe}_{3} \mathrm{O}_{21}$ (B7TFO) and $\mathrm{Bi}_{6} \mathrm{Ti}_{2.8} \mathrm{Fe}_{1.52} \mathrm{Mn}_{0.68} \mathrm{O}_{18}$ (B6TFMO) with 6 and 5 perovskite layers, respectively, were prepared by chemical solution deposition on sapphire substrates in order to increase the content of magnetic cations within 
the Aurivillius structures Fig. B.1.(b). PFM indicates that both films are ferroelectric and SQUID (superconducting quantum interference device magnetometry) magnetometry investigations demonstrate the B6TFMO films are multiferroic at room temperature. Careful microstructural analyses gives $99.5 \%$ confidence that the ferromagnetic responses originate from the parent phase. PFM under magnetic fields has given direct evidence that the ferroelectric and ferromagnetic order parameters within the $\mathrm{Bi}_{6} \mathrm{Ti}_{2.6} \mathrm{Fe}_{1.77} \mathrm{Mn}_{0.63} \mathrm{O}_{18}$ grains are magnetoelectrically coupled. This material is therefore an exciting candidate for potential use in multiferroic, magnetoelectric logic devices. 


\section{Experimental}

Thin Film Synthesis: Solutions of $\mathrm{Bi}_{7} \mathrm{Ti}_{3} \mathrm{Fe}_{3} \mathrm{O}_{21}\left(\mathrm{~m}=6\right.$; B7TFO) and $\mathrm{Bi}_{6} \mathrm{Ti}_{2.5} \mathrm{Fe}_{1.75} \mathrm{Mn}_{0.75} \mathrm{O}_{18}$ $\left(\mathrm{m}=5\right.$; B6TFMO) were prepared by dissolving $\mathrm{Bi}\left(\mathrm{NO}_{3}\right)_{3} .5 \mathrm{H}_{2} \mathrm{O}$ and $\mathrm{Ti}\left(\mathrm{OCH}_{2} \mathrm{CH}_{2} \mathrm{CH}_{2} \mathrm{CH}_{3}\right)_{4}$ in lactic acid at room temperature. $\mathrm{Fe}\left(\mathrm{NO}_{3}\right)_{3} \cdot 9 \mathrm{H}_{2} \mathrm{O}$ and $\mathrm{Mn}\left(\mathrm{C}_{5} \mathrm{H}_{7} \mathrm{O}_{2}\right)_{3}$, as appropriate, were dissolved separately in acetylacetone. When complete dissolution was achieved, the $\mathrm{Fe}^{3+}$ or $\mathrm{Fe}^{3+} / \mathrm{Mn}^{3+}$ solution was slowly dropped into the $\mathrm{Bi}^{3+} / \mathrm{Ti}^{4+}$ solution under constant stirring to prepare 0.03 moldm $^{-3}$ solutions. For all solutions, $17.5 \mathrm{~mol} \%$ excess bismuth ${ }^{41}$ was used to compensate for evaporation of bismuth during the annealing process. The films were spincoated on $c$-plane sapphire substrates by a commercial spinner (spin coater KW-4A, Chemat Technology) operating at $1000 \mathrm{rpm}$ for $30 \mathrm{~s}$. Residual organics were removed from the films by baking on a calibrated hot plate at $300 \pm 5^{\circ} \mathrm{C}$ for approximately $10 \mathrm{mins}$. Films were annealed in ambient air for 1 hour in a conventional furnace at temperatures of $850^{\circ} \mathrm{C}$. Final thicknesses of $\sim 100 \mathrm{~nm}$ and $\sim 200 \mathrm{~nm}$ were obtained for B7TFO and B6TFMO, respectively, as observed from cross-section HR-SEM measurements.

X-Ray Diffraction: XRD profiles were recorded at room temperature using a Philips Xpert PW3719 MPD diffractometer, equipped with a $\mathrm{Cu}-\mathrm{K} \alpha$ radiation source (40 kV and 35 $\mathrm{mA}$ ) and a nickel filter on the incident beam over the range $5^{\circ} \leq 2 \theta \leq 37.5^{\circ}$. To estimate the degree of $c$-axis orientation, the Lotgering factor ${ }^{61}, f$, was calculated using theoretical $(h k l)$ intensities of B7TFO and B6TFMO obtained from Crystallographica ${ }^{62}$ software.

Topography Mapping: HR-SEM images and EDX analysis spectra were obtained using a FEI Quanta 630 High Resolution Scanning Electron Microscope with attached Oxford X-Max 20 detector and Inca analysis software. A commercial atomic force microscope (MFP-3D ${ }^{\mathrm{TM}}$, Asylum Research) in AC mode, equipped with Olympus AC160TS silicon cantilevers ( $\mathrm{Al}$ reflex coated, $\sim 300 \mathrm{kHz}$ resonant frequency), was used for topography mapping of the films. Cross-sections of the films were prepared for micro-structural analysis 
using a FEI DualBeam Helios NanoLab 600i Focussed Ion Beam (FIB) (final thinning at 93 pA $30 \mathrm{kV}$, final polish $2 \mathrm{kV} 28 \mathrm{pA}$ ). Samples were gold-coated to prevent charging. Microstructural analysis was performed on the B6TFMO films using HR-TEM (Jeol 2100 transmission electron microscope; $200 \mathrm{kV}$; double tilt holder) in conjunction with selected area electron diffraction (SAED). Note that normally $\sim 10 \%$ error should be accounted for when calculating $d$-spacings from SAED due to electron optics of the instrumentation. Elemental mapping using EDX (Oxford X-Max 80 detector and Inca analysis software) over larger sample areas $\left(3.99 \mu \mathrm{m}^{2}\right.$ to $1 \mathrm{~mm}^{2}$, medium spot size, $\mathrm{x}$-ray generation area $10-30 \mathrm{~nm}$ in diameter, $200 \mathrm{~nm}$ thickness) was performed using the HR-SEM and STEM mode at the FEI Helios Nanolab. The EDX measurements were averaged over 6-7 single measurements each containing up to 1100 frames. Hence the precision of the elemental ratios is based on the standard deviation ${ }^{21}$ calculated, which ranges from $0.3 \%$ for Bi (heavy element) to $2.4 \%$ for the $\mathrm{Mn}$ and Fe ratios. This is comparable with literature under similar conditions. ${ }^{63}$

Piezoresponse Force Microscopy: Electromechanical responses of the films were measured by PFM (see Sidebar C.) using an Asylum Research MFP-3D ${ }^{\mathrm{TM}}$ AFM in contact mode, equipped with a HVA220 Amplifier for PFM using Single Frequency (drive frequency of $20 \mathrm{kHz}$ ) and Dual AC Resonance Tracking Piezoresponse Force Microscopy (DARTPFM $)^{64}$ modes. Vertical hysteresis loop measurements were obtained by switching spectroscopy PFM (SS-PFM) ${ }^{65,66}$ using a triangular step waveform (comprised of pulse DC bias voltage (60-88 V) and an AC signal (5.5 V)). The waveform was cycled twice at a frequency of $0.3 \mathrm{~Hz}$ with $68 \mathrm{AC}$ steps per waveform. PFM imaging under a magnetic field was performed using the VFM2-HV (Asylum Research High Voltage Variable Field Module (Version 2)), where rotation of the rare earth magnet allowed the maximum magnetic field intensity at the sample to be varied. In this module, the magnetic field lines are parallel along the short axis of the PFM cantilever. All images were conducted at a scan angle of $90^{\circ}$, 
where motion along this axis is parallel to the magnetic field lines and repeat measurements ( $\sim 10$ times) were performed to ensure imaging artifacts were not present. Olympus AC240TM Electrilevers, Ti/Pt coated silicon cantilevers (Al reflex coated, $70 \mathrm{kHz}$ resonant frequency, $\sim 320 \mathrm{kHz}$ contact resonance frequency) were used for PFM imaging. Note that these probes are conducting, but nonmagnetic. The Inverse Optical Lever Sensitivity of the cantilevers was calibrated according to the MFP-3D Procedural Operation 'Manualette', the system inherent background was determined using a non-piezoelectric silicon wafer and the PFM was then calibrated using $\alpha$-quartz as a reference sample. 


\section{Sidebar C. Piezoelectric Force Microscopy applied to Ferroelectric Thin Films}

Piezoresponse Force Microscopy (PFM) has emerged as a powerful technique for locally probing nanoscale phenomena in piezoelectric and ferroelectric materials on the nanometer scale. $^{67,68,69}$ PFM is based on the detection of a bias-induced piezoelectric surface deformation. ${ }^{70}$ A conductive tip is brought into contact with a piezoelectric sample surface, and the tip deflection resulting from the expansion or contraction of the sample due to the applied bias is measured (Fig. C.1.(a)).

Out-of-plane polarization is measured by recording the tip-deflection signal at the frequency of modulation. The relationship between the strain and the applied electric field in piezoelectric materials is called the 'converse piezoelectric effect' and the vertical displacement under voltage can be expressed as follows:

$$
\Delta z=\kappa\left(d_{33} V+\left(Q_{333} / t\right) V^{2}\right)
$$

where $V$ is the applied voltage, $t$ is the sample thickness, $d_{33}$ and $Q_{333}$ are the piezoelectric and electrostrictive coefficients, respectively, and $\kappa$ is a constant of proportionality, placed in the equation to indicate that the piezoelectric and electrostrictive coefficients measured using PFM are not numerically identical with the coefficients measured using (for example) IEEE (Institute of Electrical and Electronics Engineers) Standard methods on bulk specimens because of effects such as electric field distortion and substrate clamping (on thin films). $d_{33}$ is the most important component of the piezoelectric tensor for a typical vertical PFM since it couples directly into the vertical motion of the cantilever. The electrostrictive effect is quadratic and does not depend on the sign of the electric field with respect to the specimen polarization and causes only a constant effect, which is zero in the absence of an applied bias. On application of the external voltage from the tip, $\boldsymbol{V}_{t i p}=\boldsymbol{V}_{\boldsymbol{d c}}+\boldsymbol{V}_{\boldsymbol{a c}} \cos (\omega t)$, driven at 
frequency well below that of the contact resonance of the cantilever, the local piezoelectric response is detected as the first harmonic response of the tip deflection:

$$
\Delta z=d_{33} V_{d c}+d_{33} V_{a c} \cos (\omega t+\varphi)
$$

where $\varphi$, the phase of the electromechanical response of the surface, yields information on the polarization direction below the tip. Both the magnitude and sign of the displacement can be translated into images of local piezoresponse and polarization direction observed as amplitude and phase micrographs, respectively (e.g. Figs. 6(b) and (c) in the main document). When the polarization is parallel to the sample surface, the voltage applied to the tip in the lateral PFM mode couples to an in-plane surface displacement via one of the piezoelectric shear coefficients, which is translated into a torsional deflection of the cantilever. Due to the small radius of the PFM tip, the applied electric field spreads within the top layer of the sample resulting in rapidly decreasing field strength at larger sample depths. A bottom-electrode ensures a well-defined electric field distribution and thereby reproducible conditions for PFM imaging. ${ }^{71}$ In the absence of a conductive bottom electrode, part of the electric field decays in the substrate, whereas with a bottom electrode the electric field is fully applied to the film. Therefore, for thin film samples, a bottom electrode is more advantageous for PFM measurements, while larger bias values are required to image ferroelectric domains in the absence of a bottom electrode. ${ }^{72}$ As Soergel ${ }^{71}$ has explained, there is no absolute need for a back electrode and PFM images have been recorded on films of $10 \mathrm{~nm}$ thickness without a bottom electrode. However the stabilization of switched domains is greatly facilitated when a bottom electrode is present. ${ }^{72}$

The DART-PFM (Dual AC-Resonance Tracking-PFM) technique was developed by Rodriguez et $a l .{ }^{64}$ to overcome problems in electromechanical imaging of piezoelectric materials with relatively small vertical piezoresponses. The method uses the cantilever resonance frequency to boost the piezo signal in the vertical direction, while reducing 
crosstalk between changes in the sample-tip contact stiffness and the PFM signal by tracking the resonance frequency based on amplitude detection feedback. The amplitude is measured at one drive frequency below the resonance frequency and another above it. The error signal allows changes in the resonance frequency to be tracked, thereby reducing the effects of cross-talk between the PFM signal and changes in the sample-tip contact stiffness.

As well as local nanoscale imaging and polarization mapping, PFM spectroscopy modes can locally generate hysteresis loops and thereby provide information on local ferroelectric switching behaviour. ${ }^{65,66,73}$ During acquisition of a hysteresis loop in switching spectroscopy-PFM, the conducting PFM tip is fixed at a given location on the sample surface and a triangle step bias waveform is applied as a function of time (Fig. C.1.(b)). The signal has two components, a DC bias voltage which is stepped up and down on a saw-tooth profile, plus an AC signal (at much higher frequency; refer to insets in Fig. C.1.(b)) which is used to measure the displacement caused by the piezoelectric effect in the sample. The system records the amplitude and phase of this displacement signal with the "Field On" followed by the displacement signal with "Field Off". Piezoelectric hysteresis loops are thus generated with the 'Field On' and 'Field Off' (e.g. Figs. 7(a) to (d)) allowing characterization of electromechanical and structural properties of a wide range of ferroelectric materials leading to a better understanding of material functionality down to the nanoscale level. Note that the electrostrictive term in equation C.1. above is zero in the "field off" condition. The response in the "field on" state can also be affected by electrostatic interactions between the cantilever and the back electrode, which can be mistakenly interpreted as being due to electrostriction, and should thus be treated with caution.

In ferroelectric lithography, the external field is applied vertically to a ferroelectric thin film using the PFM probe, allowing complex ferroelectric patterns to be 'written' without changing the surface topography. These patterns can be 'read' after the electric field 
is removed (e.g. Figs. 7(e) to (f)), demonstrating that polarization information can be stored in the thin films and potentially encoded into rapid, energy efficient computer memory that persists even when powered-off (non-volatile random access memory).

The VFM2-HV Variable Field Module ${ }^{74}$ allows simultaneous MFM and PFM under high tip-sample voltage bias and can provide direct evidence of magnetoelectric coupling by allowing simultaneous piezoelectric force microscopy and magnetic force microscopy to locally image the coupled piezoelectric-magnetic switching. The VFM2-HV can apply static magnetic fields up to \pm 0.8 Tesla ( $\sim \mathrm{G}$ resolution), parallel to the sample plane, therefore allows observation of the effects of applying high magnetic fields in-plane while performing piezoresponse force microscopy experiments. It uses a unique design incorporating rare earth magnets to produce the magnetic field. Because of this, there is no heating or drift as the field changes, providing low-noise, high precision scanning probe measurements. Rotation of the powerful rare earth magnet allows the maximum magnetic field intensity at the sample to be varied (maximum field when rotated at $90^{\circ}$, field is turned off at 0 or $180^{\circ}$ ). Once a field value is reached, the motor is turned off and the field is maintained without residual heat, thermal drift, or mechanical vibration. 
SQUID Magnetometry: Room temperature magnetic measurements of the thin films were carried out using a Quantum Design SQUID magnetometer (Quantum Design USA; Model- MPMS XL5) under a maximum applied field of 5T (see Sidebar D. for a description of the interpretation of SQUID magnetometer responses). Before measuring any sample an appropriate demagnetization protocol was followed in order to remove any remanent magnetization. The field was set to zero field from a higher field through an oscillating field sequence. The superconducting coil of the SQUID was warmed up to room temperature to remove any trapped flux. The diamagnetic contribution from the quartz substrate was subtracted from the magnetic hysteresis loop measurements. The film weight was estimated from sample area and film thickness measurements, combined with the X-ray density to be $1.02 \times 10^{-4} \mathrm{~g}^{62}$. 
Sidebar D. Oxide Magnetism and SQUID Magnetometry

Plots of the magnetic susceptibility of typical paramagnetic, ferromagnetic and antiferromagnetic materials as a function of temperature are strongly indicative of these different types of magnetic structures, and examples are shown in figure D.1. The magnetic susceptibility $(\chi)$ is inversely proportional to temperature $(\mathrm{T})$ for paramagnetic materials (Fig. D.1.(a)). Ferromagnetic materials are paramagnetic above the Curie temperature $\left(T_{C}\right)$. However, below $\mathrm{T}_{\mathrm{C}}$ the relation between $\chi$ and $\mathrm{T}$ is complex (Green region in Fig. D.1.(b)). In case of antiferromagnetic materials below the Nèel temperature $\left(\mathrm{T}_{\mathrm{N}}\right)$, which is recognized by a sharp peak in the susceptibility curve (see Fig. D.1.(c)), the spontaneous magnetic ordering opposes the natural tendency of the magnetic moments to align parallel to the external applied field, which leads to a decrease in susceptibility as the temperature is reduced further.

Measurement of magnetic susceptibilities with high sensitivity is possible with a SQUID (Superconducting Quantum Interference Devices) magnetometer. An equivalent SQUID circuit $^{75}$ is shown in Fig. D.2. A closed superconducting loop, which consists of a pickup coil and input coil, is shown in the figure. A persistent current is generated in the superconducting loop due to the magnetic flux field measured at the pickup coil. The two Josephson tunnel junctions in SQUID which are shunted with resistors eliminate hysteresis in tunnel junction current-voltage characteristics. The output voltage across the Josephson junction appears due to the magnetic signal input at the pickup coil. The output voltage gradually changes due to the change of magnetic field for the quantum interference in Josephson junctions. Later this output signal is refined through modulation coil and converted to a magnetic moment. 
Different measurement protocols can be used. The ZFC (zero field cooled)-FC (field cooled) curve is one widely used protocol to investigate the magnetic properties as a function of temperature and bias field, and generally follows a particular procedure explained below:

1. The sample is cooled down from a high starting temperature (normally room temperature) to low temperature $(2-5 \mathrm{~K})$ without applying any magnetic field.

2. A small magnetic field (chosen from the linear region of hysteresis loop of respective sample) is applied and maintained and response moment from the sample is measured while the temperature is swept up to the starting point and down again with the same cooling rate and data accusing rate.

3. Finally the field is removed and magnetization is measured with increasing temperature from lowest temperature to the highest temperature.

Hence the final curve is made of three different parts: Zero Field Cooled (ZFC), Field Cooled (FC) and remanence curves (Fig. D.3(a)). The point at which splitting between ZFCFC curves occurs gives the transition temperatures, for example Néel $\left(T_{N}\right)$ or blocking $\left(T_{B}\right)$ temperatures, below which, the material is antiferromagnetic or ferromagnetic, and will give small or large positive remanences respectively. Another important measurement is the (MH) measurement, where the magnetization $(M)$ of the sample is measured as a function of applied magnetic field $(H)$ (Fig. D.3.(b)). For paramagnetic and diamagnetic materials, the curves obtained are straight lines through the origin with positive and negative slopes respectively. For other types of materials (ferromagnetic/ferrimagnetic/antiferromagnetic/ superparamagnetic, etc.) the $\mathrm{M}-\mathrm{H}$ measurement is nonlinear, for example giving the schematic hysteresis loop in figure D.3.(b) 


\section{Results and Discussion}

\section{(1) Structural and Compositional Analysis}

Chemical solution deposition processes were used to make $\mathrm{BiFeO}_{3}$ and $\mathrm{BiMn}_{\mathrm{x}} \mathrm{Fe}_{\mathrm{y}} \mathrm{O}_{3}$ doped $\mathrm{Bi}_{4} \mathrm{Ti}_{3} \mathrm{O}_{12}$ Aurivillius thin films on sapphire substrates. Two sols were formulated, with the intention of producing materials with $m=6$. These possessed Ti:Fe:Mn ratios of 1:1:0 and 1:0.7:0.3 respectively. Considerable (17.5 mol \%) excesses of Bi were used in both cases to suppress pyrochlore formation ${ }^{41}$. X-ray diffraction (XRD) (Fig. 1(a)) demonstrated that both sols produced Aurivillius phase type thin films, with the film lattice parameters being $a=5.468, b=5.472$ and $c=57.554 \AA$ for the first sol, evidently producing an $\mathrm{m}=6$ film - $\mathrm{Bi}_{7} \mathrm{Ti}_{3} \mathrm{Fe}_{3} \mathrm{O}_{21}$ (B7TFO). The second gave $a=5.497, b=5.415$ and $c=49.280 \AA$, clear evidence for an $\mathrm{m}=5$ film, from an average sol composition of $\mathrm{Bi}_{6} \mathrm{Ti}_{2.5} \mathrm{Fe}_{1.75} \mathrm{Mn}_{0.75} \mathrm{O}_{18}$ (B6TFMO). (Note that as a considerable excess of $\mathrm{Bi}$ is included in the sol, the minor changes in excess given by producing an $\mathrm{m}=5$, rather than an $\mathrm{m}=6$ film are not significant.) All diffraction lines could be indexed either to the relevant Aurivillius phase patterns, substrate peaks or CuKßlines from the strongest main phase peaks The thin films are predominantly $c$-axis oriented with a Lotgering factors ${ }^{61}, f$, of 0.977 for B7TFO and 0.997 for B6TFMO. The 5-layered structure for B6TFMO was confirmed by High Resolution Transmission Electron Microscopy (HR-TEM) (Fig. 1(b)) and electron diffraction (Fig. 1(c)). Note that there are no detectable lines from spinel (or indeed any other) minor phases visible in the XRD patterns of the films - the strongest (311) spinel reflections would be expected at $2 \theta=35.4^{\circ}$. However, the noise level in any XRD scan places a limit on the detectability on such minor phases and the method is intrinsically unable to detect trace levels (typically 1-3 vol \%, depending on the relative compositions of secondary and parent phases) of strongly magnetic secondary phases which may affect the overall magnetization of the 
sample. ${ }^{54}$ Clearly, detailed microstructural assessment is required for any sample of this type to exclude this as a possibility, and ideally to place a confidence level on that exclusion.

HR-SEM (high resolution scanning electron microscopy) images (Fig. 2) reveal the characteristic plate-like grain morphologies expected from Aurivillius phase materials. Multiple HR-SEM energy dispersive x-ray (EDX) surface scans (areas ranging from $900 \mu \mathrm{m}^{2}$ to $1 \mathrm{~mm}^{2}$ ) showed an average film composition of $\mathrm{Bi}_{6} \mathrm{Ti}_{2.8} \mathrm{Fe}_{1.52} \mathrm{Mn}_{0.68} \mathrm{O}_{18}$, which is slightly deficient in Fe and Mn relative to the sol. Neither a 2 hour HR-SEM-EDX area scan of a $26 \mu \mathrm{m} \times 22.6 \mu \mathrm{m}$ area $\left(120 \mu \mathrm{m}^{3}\right.$ volume), nor a STEM (scanning transmission electron microscopy) EDX examination of a $30 \mu \mathrm{m}$ long cross-section of thin film (1.2 $\mu^{3}$ volume) produced any evidence of Fe-rich regions that might indicate possible evidence of low-level minor phases. However, a 72 hour long HR-SEM-EDX data collection over a $1600 \mu^{2}$ area, followed by subtraction of the Bi L $\alpha$ from the Fe $\mathrm{K} \alpha$ and $\mathrm{Mn} \mathrm{K} \alpha$ signals produced maps which showed areas of excess Fe and Mn for the B7TFO and B6TFMO films (Figs 3(a),(b),(c)). These maps showed extremely small amounts ( $0.01 \mathrm{vol} \%)$ of $\mathrm{FeO}_{\mathrm{x}}$ oxide inclusions in B7TFO and slightly larger amounts ( 0.1 vol\%) of a $\mathrm{Fe}_{\mathrm{x}} \mathrm{Mn}_{\mathrm{y}} \mathrm{O}_{\mathrm{z}}$ phase in the B6TFMO with Mn:Fe ratio of 1.13:1 and a size of $\sim 350 \mathrm{~nm}$. HR-STEM-EDX examination of the $\mathrm{Fe}_{\mathrm{x}} \mathrm{Mn}_{\mathrm{y}} \mathrm{O}_{\mathrm{z}}$ inclusions demonstrated a composition of $\mathrm{Mn}_{0.53} \mathrm{Fe}_{0.47} \mathrm{O}$. HR-TEM/SAED (selected area electron diffraction) (Fig. 3(e)) indicates a cubic structure with a lattice parameter of $4.4 \AA$, that closely corresponds to that of a rock-salt-structure ${ }^{76}$. Magnetite $\left(\mathrm{Fe}_{3} \mathrm{O}_{4}\right)$ and Jacobsite $\left(\mathrm{MnFe}_{2} \mathrm{O}_{4}\right)$ structures can be excluded since the measured Mn:Fe ratio of 1.13:1 does not fit the compositions of these phases. Additionally, the space group Fd-3m (227) for Magnetite and Jacobsite does not fit the electron diffraction pattern obtained for the inclusions. The bixbyite phase $\left(\mathrm{Mn}_{2-2 \mathrm{x}} \mathrm{Fe}_{2 \mathrm{x}} \mathrm{O}_{3}\right.$ where $\left.\mathrm{x}=0.4-0.6\right)$ can also be discounted as the Ia-3 (206) spacegroup also demonstrates a different electron diffraction pattern from that obtained. Neither the angles of the reflections nor the lattice parameters of Fd-3m (227) / Ia- 
3 (206) fit in any direction to the electron diffraction pattern of the inclusions. A composition closely related to that of the inclusions, $\mathrm{Mn}_{0.56} \mathrm{Fe}_{0.44} \mathrm{O}$ (Mangano Wüstite), has a cubic lattice with the space group Fm-3m $(225)^{76,77}$. It should be noted that this rock-salt-structured composition is non-ferromagnetic, and antiferromagnetic at low temperatures with a Nèel point of $\sim 150 \mathrm{~K}^{77}$. The electron diffraction pattern captured for the $\mathrm{Mn}_{0.53} \mathrm{Fe}_{0.47} \mathrm{O}$ inclusions fits simulations of this space group along the (110) direction perfectly ${ }^{78}$ assuming micro/nano-twinning ${ }^{79,80}$ causes reflections at half positions. This assumption is strengthened by the observations in TEM dark field mode (Fig. 4). In this technique the unscattered or zero order beam is excluded. Only electrons diffracted on crystal planes contribute to the formed image. Fig. 4 shows clearly that different diffraction spots in the summary SAED (Fig. 4(a)) stem from different micro/nano-crystals. In particular three regions nearly parallel to the substrate surface are discernible in different distances. Viewed from the substrate the bottom part is most visible in Fig. 4(a), the top part in Fig. 4(e) and the interface in-between in Fig. 4(f). A second random twinning in horizontal direction is also observable within the inclusion which is illuminated by the complimentary highlighting of different crystals in Figs 4(d) and (g).

Also visible in these surface HR-SEM-EDX maps were larger areas, similar in shape to the Aurivillius grains, where the Fe content slightly exceeded the surrounding grains. Detailed cross-sectional HR-TEM/SAED and HR-STEM-EDX examination of these grains (Fig. 3(f),(g)) showed that they were $m=5$ Aurivillius-structured grains possessing a higher Fe content than the average of the film composition determined by surface area HR-SEMEDX. A compositional survey by cross-section HR-STEM-EDX of 55 individual Aurivillius grains within the B6TFMO sample, followed by normalization of the Ti:Fe:Mn ratios to give 5 octahedral B-site cations, showed a strong relationship between the Fe and Ti contents in these grains, with the Mn content being less strongly dependent on the Ti B-site composition 
(Fig. 5(a)). The range of grain compositions (ranging from Ti:Fe:Mn =3.38:1.14:0.48 to 2.18:2.16:0.66) is much greater than the observed precision of the EDX measurement technique ( 0.3 to $2.4 \%$ ) and spans both the average compositions determined from the sol and the area EDX scan noted above. This graph helps to explain why a sol which was set-up to deliver an $\mathrm{m}=6$ structure could produce an $\mathrm{m}=5$ structure without large amounts of second phase appearing in the film. Mn is well-known for taking variable valency from 3-4 in perovskite oxide materials ${ }^{81}$, and permitting non-stoichiometry to exist. As the $\mathrm{Fe}^{3+}$ replaces $\mathrm{Ti}^{4+}$, it is suggested that $\mathrm{Mn}^{3+}$ is progressively oxidized to $\mathrm{Mn}^{4+}$, to maintain charge balance. In this case, the proportion converted to the higher valence state is easily calculated and is plotted in Fig. 5(b). It is interesting to note that, while the Fe and Ti $B$-site compositions are strongly inter-dependent, the Mn composition within these grains does not vary systematically with either, averaging around 0.63 , and the maximum Fe content of the $B$-site, at 1.77 , occurs when all of the $\mathrm{Mn}^{3+}$ is converted to $\mathrm{Mn}^{4+}$. It is believed that this has important consequences for the magnetic properties of the grains, as will be discussed below.

\section{(2) Ferroelectric Characterization}

Piezoresponse force microscopy (PFM) $)^{68,73,82,83,84}$ was used to investigate the local electromechanical properties of the films at room temperature. Single frequency PFM imaging experiments demonstrate that both sets of the films are piezoelectric at room temperature, with higher piezoresponses in the lateral direction $(25 \mathrm{pm} / \mathrm{V}$ for B7TFO \& 19 $\mathrm{pm} / \mathrm{V}$ for B6TFMO) compared with those measured in the vertical direction (3 pm/V for B7TFO \& $8 \mathrm{pm} / \mathrm{V}$ for B6TFMO) (Fig. 6). As these films are preferentially $c$-axis oriented, most of the grains have their crystallographic $a$-axis lying in the lateral plane of the film. The major polarization vector for Aurivillius phase materials is along the $a$-axis ${ }^{85}$ and as a result, the single frequency lateral PFM images demonstrate greater piezoresponses than the single 
frequency vertical PFM images, which given the low piezoresponses, topography cross-talk is likely also to be contributing to the images obtained. Since the films are comprised of some $a$-axis oriented grains as well as $c$-axis oriented grains (Fig. 1(a)), a vertical PFM response arises from $a$-axis oriented grains which are tilted out-of-plane and therefore are accessible to probing by vertical PFM. There is a minor polarization along the $c$-axis for Aurivillius phases with odd numbers of perovskite layers. Therefore, the difference between lateral and vertical PFM responses for the B6TFMO $(\mathrm{m}=5)$ films is less than that of the B7TFO $(\mathrm{m}=6)$ films. We employed the DART-PFM mode (Fig. 7) to intensify the imaging of the weaker out-of-plane component and reduce effects of topography cross-talk. Investigations of the local room temperature ferroelectric switching behavior in the films by Vertical DART-PFM switching spectroscopy measurements in the absence of an applied DC bias are presented for B7TFO in Fig. 8(a) and (b), and for B6TFMO in Fig. 8(c) and (d), where $180^{\circ}$ ferroelectric switching is clearly demonstrated for both types of film.

Ferroelectric polarization reversal over areas of the B6TFMO film could be achieved by applying an applied voltage of up to $70 \mathrm{~V}$ vertically to an area of the samples via the PFM tip (in a "write" step). Given that the insulating substrate is $400 \mu \mathrm{m}$ thick, this would correspond

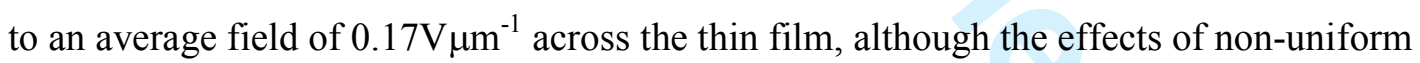
field spreading from the tip imply that the field within the film will be considerably higher than this. The written areas could be detected by a subsequent PFM scan ("read" step), as is demonstrated in Fig. 8(e) and (f). Tests conducted over an 8 hour period demonstrated that the films retained polarization for this period of time.

\section{(3) SQUID Magnetometry Investigations}

The magnetic behavior of the B6TFMO and B7TFO thin films were investigated using SQUID magnetometry from 2 to $300 \mathrm{~K}$. A strong ferromagnetic signature was observed for 
the B6TFMO samples, as is evident from SQUID magnetization measurements as a function of magnetic field (Fig. 9(a)) and temperature (Fig. 9(b)), whereas a clear antiferromagnetic behavior was observed in case of B7TFO (Fig. 9(d)). The average sample thickness was calculated after measuring at $\sim 200$ cross section points across the sample piece. Additionally we take in to account that $2.44 \%$ area of the substrate was not covered by the Aurivillius phase thin film due to pore formation, (inset figure Fig. 9(a)). Accordingly, the saturation magnetization $\left(M_{\mathrm{S}}\right)$ measured for Aurivillius phase B6TFMO is calculated to be $0.74 \mathrm{emu} / \mathrm{gm}$ with remanent magnetization $\left(M_{\mathrm{r}}\right)$ of $0.022 \mathrm{emu} / \mathrm{g}\left(0.18 \mathrm{emu} / \mathrm{cm}^{3}\right)$ and coercivity $\left(\mu_{0} \mathrm{H}_{\mathrm{c}}\right)$ of $7 \mathrm{mT}$ at $300 \mathrm{~K}$. The coercivity and remanence increase gradually as temperature decreases (Table 9(c)). ZFC (zero field cooled) - FC (field cooled) measurements (Fig. 9(b)) were performed to investigate the magnetization behavior of the B6TFMO sample as a function of temperature. A relatively low field of $10 \mathrm{mT}$ was applied for these measurements. The clear split between the ZFC-FC curves demonstrates the ferromagnetic nature of the sample since otherwise the ZFC-FC lines would normally coincide ${ }^{86}$ with each other. The non-substituted compound, $\mathrm{B} 7 \mathrm{TFO}\left(\mathrm{Bi}_{7} \mathrm{Ti}_{3} \mathrm{Fe}_{3} \mathrm{O}_{21}\right)$, demonstrates an antiferromagnetic Nèel temperature at $190 \mathrm{~K}$ and a magnetic transition to weak ferromagnetism below 35K (Fig. 9(d)) in accordance with earlier reports ${ }^{50}$. The antiferromagnetic secondary phase $\mathrm{Mn}_{0.53} \mathrm{Fe}_{0.47} \mathrm{O}$ observed in B6TFMO by HR-SEM is reported to have a Nèel Temperature at $\sim 150 \mathrm{~K}^{77}$. However there is no $150 \mathrm{~K}$ magnetic transition detected in the measurement magnetization vs. temperature measurement (MT) for B6TFMO. Rather, the ZFC-FC curves are well separated below $350 \mathrm{~K}$ which strengthens the evidence for B6TFMO being ferromagnetic, with a $\mathrm{T}_{\mathrm{C}}$ greater than $350 \mathrm{~K}$. Further it is observed that the FC curve of B6TFMO drops down at $190 \mathrm{~K}$ and again increases sharply below $35 \mathrm{~K}$ which is similar in nature with the MT behavior of B7TFO (Fig. 9(d)). This non-monotonic behavior of the FC curve for B6TFMO can be explained as follows. It is most likely that a significant part of the parent B6TFMO phase has 
been modified to become ferromagnetic, with the remainder being antiferromagnetic in the same way as unmodified B6TFO ${ }^{57,59,87,88}$. The variation of magnetization of B6TFMO as a function of temperature (Fig. 9(b)) is thus non-monotonic in nature due to the influence of a proportion of grains behaving as would be expected from the unmodified parent antiferromagnetic B6TFO phase ${ }^{87,88}$. In the magnetic hysteresis loop measurement (Fig. 10), the B6TFMO film shows a saturation magnetization $(2.19 \mathrm{emu} / \mathrm{gm}$ at $2 \mathrm{~K}$ temperature-5T field) which is substantially higher than the unsubstituted B7TFO phase $(0.2 \mathrm{emu} / \mathrm{gm}$ at $5 \mathrm{~K}$ temperature-5T field). For antiferromagnetic materials the remanence magnetization is naturally near zero (ideally zero) as the opposite spins cancels out each other. The observation of both high remanence and an increase of remanence and coercivity of B6TFMO with decrease of temperature in B6TFMO strongly supports ferromagnetism in this material.

\section{(4) Evidence for Magnetoelectric Multiferroic Coupling in B6TFMO}

Direct evidence of magnetoelectric multiferroic coupling was sought by performing piezoresponse force microscopy under a variable magnetic field to locally image any coupled piezoelectric-magnetic switching. Single frequency lateral PFM was performed on the B6TFMO sample, as shown in Fig. 11(c) and (d). On application of an in-plane magnetic field of $+250 \mathrm{mT}$, which is above that of the coercive field of the B6TFMO sample (Fig. 9(c)), two situations, (i) the emergence of in-plane piezoelectric domains (blue and green circles) and (ii) piezoelectric domain switching (red and orange circles) were observed by means of in-plane PFM imaging (Fig. 11(e) and (f)). Vertical PFM in single frequency mode was also performed in the same area; however the vertical response is much lower than the in-plane response and mostly at noise level. This is because of the fundamental fact that most of the polarization (and hence piezoresponse) in these highly-oriented Aurivillius grains lies 
in the plane of the sample. Occasionally some vertical response is observed, which is due to the presence of some grains which have a significant out-of-plane orientation. There are no obvious vertical PFM domains in the regions where domain evolution was observed in the lateral PFM scans (green and blue circles). Since the torsional twist of the cantilever used for PFM scanning is parallel to that of the magnetic field lines, piezoelectric domain formation and polarization switching is solely induced by the external magnetic field and a coupling of the electric and magnetic order parameters within the B6TFMO grains. Polarization reversal due to the action of the imaging ac field is unlikely to occur at the imaging frequency. ${ }^{89}$ Induction of piezoelectric polarization reversal by application of a magnetic field in the positive direction $(+250 \mathrm{mT})$ was also observed by vertical PFM imaging of the out-of-plane piezoresponses. Vertical DART PFM imaging (Fig. 12(e), (f)) indicated (via piezoelectric signal phase inversion) areas of local ferroelectric domain switching / polarization reversal due to the magnetic field. When a magnetic field of $250 \mathrm{mT}$ was applied in the opposite (negative) direction, additional areas exhibiting polarization inversion were obtained (Fig. 12(h),(i)). With the data obtained, it is not possible to distinguish between 180 and 90 degree ferroelectric polarisation switching under the application of the magnetic field. Magnetoelectric switching has been observed in both lateral (Fig. 11) and vertical (Fig. 12) PFM experiments. Given that the orientations of the specific grains exhibiting the effects are indeterminate, the details of the relative crystallographic orientation of ferroelectric and magnetic polarisation directions will be the subject of further work. Ideally, we need single crystal epitaxial films to do that, which will be grown by AVD (atomic vapour deposition). The switching regions were approximately $250 \mathrm{~nm}$ in size, clearly related to the Aurivillius grains, of which they were a small fraction of the total number (average change in polarization was 4\% for Fig. 11 and 7\% for Fig. $\mathbf{1 2}^{90}$ ) and widely dispersed throughout the film. The mechanism by which the coupling occurs is not obvious from these experiments. 
Evans et al. ${ }^{36}$ put forward a strain-mediated coupling mechanism for their observations of ferroelectric domain switching in $\left[\mathrm{Pb}\left(\mathrm{Zr}_{0.53} \mathrm{Ti}_{0.47}\right) \mathrm{O}_{3}\right]_{0.6}-\left[\mathrm{Pb}\left(\mathrm{Fe}_{0.5} \mathrm{Ta}_{0.5}\right) \mathrm{O}_{3}\right]_{0.4}$ on application of a magnetic field. This direct observation of the switching and formation of a ferroelectric polarization induced by a change in magnetic field within a single phase is significant as it provides strong evidence that within the B6TFMO thin film sample there exists Aurivillius phases at the nanoscale, where ferromagnetic-ferroelectric order parameters are coupled within a single phase and are multiferroic at room-temperature. It is proposed that the dispersion of the grains showing the multiferroic switching reflects the fact that these are the grains with the highest levels of Fe/Mn present, probably with a composition at around $\mathrm{Bi}_{6} \mathrm{Ti}_{2.6} \mathrm{Fe}_{1.77} \mathrm{Mn}_{0.63} \mathrm{O}_{18}$, for which the majority of the Mn will be present as $\mathrm{Mn}^{4+}$. This conclusion is supported by the magnetic susceptibility measurements, where a proportion of the film is apparently ferromagnetic and a proportion antiferromagnetic, as in unmodified B6TFO.

\section{(5) Statistical Treatment of Microstructural Analysis}

The appearance of ferromagnetism in our B6TFMO sample is intriguing. As described above, we have conducted extremely detailed microstructural analysis and have seen very small amounts $(0.1 \%)$ of a $\mathrm{Mn}_{0.53} \mathrm{Fe}_{0.47} \mathrm{O}$ phase having the rock-salt structure, which is antiferromagnetic $<150 \mathrm{~K}$, but have detected no ferromagnetic phases. However, it is impossible to be $100 \%$ certain that there are absolutely no ferromagnetic spinel grains (e.g. $\left.\mathrm{Fe}_{3} \mathrm{O}_{4}\left(\mathrm{M}_{\mathrm{r}}=20 \mathrm{emu} / \mathrm{g}, \mathrm{M}_{\mathrm{s}}=90 \mathrm{emu} / \mathrm{g}\right)^{91} ; \mathrm{MnFe}_{2} \mathrm{O}_{4}\left(\mathrm{M}_{\mathrm{r}}=18 \mathrm{emu} / \mathrm{g}, \mathrm{M}_{\mathrm{s}}=80 \mathrm{emu} / \mathrm{g}\right)^{92}\right)$ in the sample, which just did not happen to be seen in the microstructural surveys. In order to check if the ferromagnetic response might be due to magnetic second (or impurity) phase inclusions, we have performed a statistical analysis of the upper bound of the density of magnetic inclusions, such as $\mathrm{Fe}_{3} \mathrm{O}_{4}$ spinel particles, that might be expected in the sample. 
A number of measurements were performed to search for possible inclusions in the sample, and for each of them the scanned volume and the minimal detectable particle size was recorded. This data is summarized in Table 1. The EDX surface scans have been recorded in $1024 \times 886$ pixels per map. For measurement 1 (area of $10,000 \mu \mathrm{m}^{2}$ ) the detection limit for inclusions is $1 \times 1$ pixel. For smaller areas the smallest detectable size increases to $2 \times 2$ and $3 \times 3$ pixels because the EDX interaction volume is mainly dependent on the beam energy, and hence stays constant. Its size is independent of how finely the area is rastered. Recording an average of $4.4 \times 10^{9}$ counts over 72 hours improved the signal-to-noise-ratio (SNR) by a factor of 67 compared to a standard scan with an average of $1 \times 10^{6}$ total counts. This was one of the two key factors in the ability to record inclusions down to $0.01 \mathrm{vol} \%$. The other key factor was the subtraction of the background Fe that was bound to the main phase, hence making the excess Fe visible (Figure $3 \mathrm{a}-\mathrm{c}$ ). Based on the measurements performed, we can exclude the possibility that grains of diameters between $5 \mathrm{~nm}$ and about $5 \mu \mathrm{m}$ are responsible for the observed remanence.

Let us assume that in measurement number $k$ a sample volume $V_{k}$ is scanned for inclusions with a diameter larger than some minimal detectable diameter $d_{k}$. If no inclusions are found in this measurement, then we can conclude that the density of inclusions $\rho_{k}$ larger than $d_{k}$ has an upper bound given by $\rho_{k}<5.3 / V_{k}$ with a confidence level of $99.5 \%$. This then allows us to put an upper limit on the volume fraction $\phi_{k}$ of expected magnetic inclusions in the sample within a diameter interval $\left[d_{k}, d_{k-1}\right]$, where $d_{k-1}$ is the smallest diameter detectable in a previous measurement with less resolution. We calculate $\phi_{k}$ under the assumption that inclusions are spherical (cylindrical) for diameters less than the sample thickness $d_{s}=0.2 \mu \mathrm{m}$ and the corresponding values are given in Table 1. Based on this analysis we are able to calculate an upper bound for the contributions of impurity inclusions to the remanence magnetization $M_{r}$. As we do not observe such magnetic inclusions, and 
therefore do not know their potential chemical properties, we assume as a worst case scenario that their magnetic properties are comparable to $\mathrm{Fe}_{3} \mathrm{O}_{4}$, which is the strongest known candidate impurity in the material system under consideration. We thus assume that inclusions with diameters $<5 \mathrm{~nm}$ are not ferromagnetic, inclusions of diameters between 5 and $20 \mathrm{~nm}$ contribute $<10 \mathrm{emu} / \mathrm{g}^{93}$ to the remanence of the material and all other inclusions are assumed to contribute ca $20 \mathrm{emu} / \mathrm{g}$, comparable with the bulk value for $\mathrm{Fe}_{3} \mathrm{O}_{4} \cdot{ }^{94}$ Using these upper estimates, we calculate an upper limit for the contribution from undetected impurity inclusions to the remanence in our sample. This is done in the column denoted by $M_{r, k}$ in Table 1. The maximum $M_{r, k}$ is $M_{r, 6} \approx 2.8 \mathrm{memu} / \mathrm{g}$. From this analysis we therefore finally conclude that the contribution to the remanence from unobserved inclusions in the size range below $5 \mu \mathrm{m}$ is less than $2.8 \mathrm{memu} / \mathrm{g}$ with a confidence level better than $99.5 \%$. The details of the analysis and the mathematics behind this analysis will be the subject of a separate publication. It is particularly interesting that the B7TFO film showed no room temperature ferromagnetic response, in spite of being made in a similar way to the B6TFMO film, and possessing a slightly larger number of magnetic cations/unit volume $\left(3.5 \mathrm{Fe}\right.$ ions $/ \mathrm{nm}^{3}$ for B7TFO vs. $3.3 \mathrm{Fe} / \mathrm{Mn}$ ions $/ \mathrm{nm}^{3}$ for a B6TFMO grain with a composition $\left.\mathrm{Bi}_{6} \mathrm{Ti}_{2.6} \mathrm{Fe}_{1.77} \mathrm{Mn}_{0.63} \mathrm{O}_{18}\right)$. The $\mathrm{Mn}$ ions are clearly making a major contribution to the ferromagnetic response in this material, and the fact that the multiferroic switchability is confined to a small number of grains strongly suggests that it is those grains with the highest $\mathrm{Fe} / \mathrm{Mn}$ content that are responsible for the effect, and for these the manganese is probably present as $\mathrm{Mn}^{4+}$, which possesses 3 unpaired spins in its $\mathrm{t}_{2 \mathrm{~g}} \mathrm{~d}$-orbitals, with no electrons in its $\mathrm{e}_{\mathrm{g}}$ orbitals when coordinated in the perovskite geometry. By contrast, high-spin $\mathrm{Fe}^{3+}$ possesses 2 unpaired electrons in its $\mathrm{e}_{\mathrm{g}}$ orbitals. The parent compound, B6TFO has been reported $^{59,87,88}$ to be antiferromagnetic with a magnetic transition to weak ferromagnetism below $65 \mathrm{~K}$. 
The mechanism involving ligand orbitals to facilitate coupling between metal electrons is referred to as super-exchange. According to the Goodenough-Kanamori rule ${ }^{95}$, super-exchange interactions are antiferromagnetic where virtual electron transfer is between overlapping orbitals that are each half-filled. The adjacent metal ions couple with their spins antiparallel, with equal numbers of the two arrangements so that there is no resultant magnetization in the absence of a magnetic field. On the other hand, the GoodenoughKanamori rule predicts that super-exchange interactions are strong and ferromagnetic when virtual electron transfer is from a half-filled orbital to an empty orbital. The electron spins of each of the atoms couple strongly together to form a resultant unit cell magnetic moment in an applied magnetic field which remains when the external field is removed. Hence, the Goodenough-Kanamori rule ${ }^{95}$ for superexchange between the half-filled $e_{g}$ orbitals of highspin $\mathrm{Fe}^{3+}$ via the oxygen p-orbitals in the perovskite blocks is expected to give a strong antiferromagnetic interaction and would explain the antiferromagnetic behavior we observed in B7TFO (and reported in the literature for B6TFO). However, if we introduce $\mathrm{Mn}^{4+}$ into the structure, the $\mathrm{e}_{\mathrm{g}}$ orbital is always empty. In this case, the Goodenough-Kanamori rule states that the super-exchange interaction via the oxygen p-orbitals to an empty $\mathrm{e}_{\mathrm{g}}$ orbital should lead to ferromagnetism. Therefore a ferromagnetic interaction between the vacant $\mathrm{Mn}^{4+} e_{\mathrm{g}}$ orbital and the filled $\mathrm{Fe}^{3+} e_{\mathrm{g}}$ orbital $^{95}$ within the Aurivillius phase structure is likely to cause the observed ferromagnetism in the manganese-substituted B6TFMO samples.

\section{Conclusions}

In conclusion, thin film samples of the $m=6$ and $m=5$ Aurivillius compounds containing $\mathrm{Fe}$ and $\mathrm{Fe} / \mathrm{Mn}$ ions were grown on $c$-plane sapphire substrates by chemical solution deposition displaying typical Aurivillius phase grain morphologies and with no spinel secondary phases visible in the XRD patterns. PFM images demonstrate that the films are piezoelectric and 
ferroelectric at room temperature with the major polarization vector in the lateral plane of the films. SQUID measurements demonstrated antiferromagnetism in B7TFO ( $\left.\mathrm{T}_{\mathrm{N}} 190 \mathrm{~K}\right)$, but B6TFMO samples demonstrate in-plane ferromagnetic hysteresis between 2 to $300 \mathrm{~K}$. A thorough microstructural phase analysis performed on the B6TFMO thin films showed no traces of ferromagnetic inclusions and a statistical analysis based on the volumes inspected placed a confidence of $99.5 \%$ that the observed ferromagnetism was not coming from unobserved ferromagnetic grains of spinel. Direct evidence for magnetic-field-induced ferroelectric domain switching at the nanoscale in a single phase magnetoelectric has been presented. The body of evidence reported here suggests that the higher Fe/Mn content grains with a composition of around $\mathrm{Bi}_{6} \mathrm{Ti}_{2.6} \mathrm{Fe}_{1.77} \mathrm{Mn}_{0.63} \mathrm{O}_{18}$ are single phase room temperature magnetoelectric multiferroics. An explanation for the effect has been given based upon the Goodenough-Kanamori rule for super-exchange and the Mn largely being present as $\mathrm{Mn}^{4+}$. As they stand, these materials have rather low ferromagnetic bulk magnetisation values, but we have seen that the ferromagnetic component of the films is only a proportion of the whole, and with further work they could be optimized to increase the total volume of ferromagnetic phase, in which case they may find application to a wide range of new or improved devices and potentially meet future industry requirements in high density memory applications. In any case, we believe they provide important pointers for the future development of room temperature ferroelectric/ferromagnetic multiferroics. Clearly, further work is now required which will include the direct measurement of the compositions of the multi-ferroically switchable grains and $\mathrm{x}$-ray photoelectron spectroscopy to determine Mn oxidation states, as well as the development of synthetic techniques to develop thin films in which all grains possess a composition around $\mathrm{Bi}_{6} \mathrm{Ti}_{2.6} \mathrm{Fe}_{1.77} \mathrm{Mn}_{0.63} \mathrm{O}_{18}$, for which it is expected that higher remanent magnetizations will be achieved. Further work will also include the development of films onto back electrodes which can be used for direct magnetodielectric measurements. 


\section{Acknowledgements}

The support of Science Foundation Ireland (SFI) under the FORME Strategic Research Cluster Award number 07/SRC/I1172, Starting Investigator Research Grant (09/SIRG/I1621) and SFI 09/SIRG/I1615 is gratefully acknowledged. The authors acknowledge ICGEE (International Centre for Graduate Education in micro \& nano Engineering) for funding Nitin Deepak's PhD. The authors would like to thank Asylum Research for their complimentary temporary provision of High Voltage Variable Field Module. 


\section{References}

1. "Assessment of the Potential and Maturity of Selected Emerging Research Memory Technologies Workshop \& ERD (Emerging Research Devices) /ERM Working Group Meeting (April 6-7 2010)," (2010).

2. "Emerging Research Materials, INTERNATIONAL TECHNOLOGY ROADMAP FOR SEMICONDUCTORS, 2009 Edition," (2009).

3. C. N. R. Rao, A. Sundaresan, andR. Saha, "Multiferroic and Magnetoelectric Oxides: The Emerging Scenario," J. Phys. Chem. Lett. , 3[16] 2237-46 (2012).

4. M. Bibes, "Nanoferronics is a winning combination," Nat Mater, 11[5] 354-57 (2012).

5. L. Wang, D. Wang, Q. Cao, Y. Zheng, H. Xuan, J. Gao, andY. Du, "Electric control of magnetism at room temperature," Sci. Rep., 2 (2012).

6. R. Ramesh, "Magnetoelectrics: Making metallic memories," Nat. Nanotechnol., 5[11] 761$62(2010)$.

7. J. F. Scott, "Data storage: Multiferroic memories," Nat. Mater., 6[4] 256-57 (2007).

8. C. A. F. Vaz, J. Hoffman, C. H. Ahn, andR. Ramesh, "Magnetoelectric Coupling Effects in Multiferroic Complex Oxide Composite Structures," Adv. Mater., 22[26-27] 2900-18 (2010).

9. N. A. Hill, "Why Are There so Few Magnetic Ferroelectrics?," J. Phys. Chem. B, 104[29] 6694-709 (2000).

10. G. Catalan and J. F. Scott, "Physics and Applications of Bismuth Ferrite," Adv. Mater., 21[24] 2463-85 (2009).

11. Y.-H. Chu, L. W. Martin, M. B. Holcomb, M. Gajek, S.-J. Han, Q. He, N. Balke, C.-H. Yang, D. Lee, W. Hu, Q. Zhan, P.-L. Yang, A. Fraile-Rodriguez, A. Scholl, S. X. Wang, andR. Ramesh, "Electric-field control of local ferromagnetism using a magnetoelectric multiferroic," Nat Mater, 7[6] 478-82 (2008). 
12. S. Lee, W. Ratcliff, II, S.-W. Cheong, andV. Kiryukhin, "Electric field control of the magnetic state in $\mathrm{BiFeO}[\mathrm{sub} 3]$ single crystals," Applied Physics Letters, 92[19] 192906 (2008).

13. D. Lebeugle, D. Colson, A. Forget, M. Viret, A. M. Bataille, andA. Gukasov, "ElectricField-Induced Spin Flop in BiFeO_\{3\} Single Crystals at Room Temperature," Physical Review Letters, 100[22] 227602 (2008).

14. N. A. Lomanova, V. G. Semenov, V. V. Panchuk, andV. V. Gusarov, "Structural changes in the homologous series of the Aurivillius phases $\mathrm{Bi}_{\mathrm{n}+1} \mathrm{Fe}_{\mathrm{n}-3} \mathrm{Ti}_{3} \mathrm{O}_{3 \mathrm{n}+3}$," J. Alloys Compd., 528[0] 103-08 (2012).

15. C. H. Macgillavry, G. D. Rieck, andK. Lonsdale, "International Tables for Crystallography, Volume III, Physical and Chemical Tables." The International Union of Crystallography by The Kynoch Press: Birmingham, England, (1968).

16. J. S. Kasper and K. Lonsdale, "International Tables for X-Ray Crystallography, Volume II, Mathematical Tables." The International Union of Crystallography by The Kynoch Press: Birmingham, England, (1972).

17. M. Bibes, J. E. Villegas, andA. Barthélémy, "Ultrathin oxide films and interfaces for electronics and spintronics," Advances in Physics, 60[1] 5-84 (2011).

18. A. Roy, R. Gupta, andA. Garg, "Multiferroic Memories," Advances in Condensed Matter Physics, 201212 (2012).

19. K. E. Sickafus, J. M. Wills, andN. W. Grimes, "Structure of Spinel," J. Am. Ceram. Soc., 82[12] 3279-92 (1999).

20. C. A. F. Vaz, "Electric field control of magnetism in multiferroic heterostructures " $J$. Phys.: Condens. Matter 24[3] 333201 (2012).

21. A. P. Pyatakov and A. K. Zvezdin, "Magnetoelectric and Multiferroic Media," PhysicsUspekhi, 55[6] 557--81 (2012). 
22. J. F. Scott, "Applications of magnetoelectrics," J. Mater. Chem., 22[11] 4567-74 (2012).

23. L. W. Martin, S. P. Crane, Y. H. Chu, M. B. Holcomb, M. Gajek, M. Huijben, C. H. Yang, N. Balke, andR. Ramesh, "Multiferroics and magnetoelectrics: thin films and nanostructures," J. Phys.: Condens. Matter, 20434220 (2008).

24. W. Eerenstein, N. D. Mathur, andJ. F. Scott, "Multiferroic and magnetoelectric materials," Nature, 442[7104] 759-65 (2006).

25. M. Fiebig, "Revival of the Magnetoelectric Effect," J. Phys. D: Appl. Phys., 38 R123-R52 (2005).

26. J. Ryu, S. Priya, A. V. Carazo, K. Uchino, andH.-E. Kim, "Effect of the Magnetostrictive Layer on Magnetoelectric Properties in Lead Zirconate Titanate/Terfenol-D Laminate Composites," J. Am. Ceram. Soc., 84[12] 2905-08 (2001).

27. F. Zavaliche, T. Zhao, H. Zheng, F. Straub, M. P. Cruz, P. L. Yang, D. Hao, andR. Ramesh, "Electrically Assisted Magnetic Recording in Multiferroic Nanostructures," Nano Lett., 7[6] 1586-90 (2007).

28. T. H. E. Lahtinen, K. J. A. Franke, andS. van Dijken, "Electric-field control of magnetic domain wall motion and local magnetization reversal," Sci. Rep., 2 (2012).

29. V. J. Folen, G. T. Rado, andE. W. Stalder, "Anisotropy of the Magnetoelectric Effect in $\mathrm{Cr}_{2} \mathrm{O}_{3}$," Phys. Rev. Lett., 6[11] 607-08 (1961).

30. T. Kimura, Y. Sekio, H. Nakamura, T. Siegrist, andA. P. Ramirez, "Cupric oxide as an induced-multiferroic with high-TC," Nat. Mater., 7[4] 291-94 (2008).

31. T. Kimura, T. Goto, H. Shintani, K. Ishizaka, T. Arima, andY. Tokura, "Magnetic control of ferroelectric polarization," Nature, 426[6962] 55-58 (2003).

32. E. Ascher, H. Rieder, H. Schmid, andH. Stossel, "Some Properties of Ferromagnetoelectric Nickel-Iodine Boracite, $\mathrm{Ni}_{3} \mathrm{~B}_{7} \mathrm{O}_{13} \mathrm{I}, "$ J. Appl. Phys., 37[3] 140405 (1966). 
33. D. Higashiyama, S. Miyasaka, N. Kida, T. Arima, andY. Tokura, "Control of the ferroelectric properties of $\mathrm{DyMn}_{2} \mathrm{O}_{5}$ by magnetic fields," Phys. Rev. B, 70[17] 174405 (2004).

34. C.-W. Nan, M. I. Bichurin, S. Dong, D. Viehland, andG. Srinivasan, "Multiferroic magnetoelectric composites: Historical perspective, status, and future directions," $J$. Appl. Phys., 103[3] 031101 (2008).

35. Y. Kitagawa, Y. Hiraoka, T. Honda, T. Ishikura, H. Nakamura, andT. Kimura, "Low-field magnetoelectric effect at room temperature," Nat. Mater., 9[10] 797-802 (2010).

36. D. M. Evans, A. Schilling, A. Kumar, D. Sanchez, N. Ortega, M. Arredondo, R. S. Katiyar, J. M. Gregg, andJ. F. Scott, "Magnetic switching of ferroelectric domains at room temperature in multiferroic PZTFT," Nat. Commun., 41534 (2013).

37. B. Aurivillius, "Mixed Bismuth Oxides with Layer Lattice II. Structure of $\mathrm{Bi}_{4} \mathrm{Ti}_{3} \mathrm{O}_{12}$," Ark. Kemi., 1499 (1949).

38. S. Patri, R. Choudhary, andB. Samantaray, "Studies of structural, dielectric and impedance properties of $\mathrm{Bi}_{9} \mathrm{Fe}_{5} \mathrm{Ti}_{3} \mathrm{O}_{27}$ ceramics," J. Electroceram., 20[2] 119-26 (2008).

39. P. F. Zhang, N. Deepak, L. Keeney, M. E. Pemble, andR. W. Whatmore, "The structural and piezoresponse properties of c-axis-oriented Aurivillius phase $\mathrm{Bi}_{5} \mathrm{Ti}_{3} \mathrm{FeO}_{15}$ thin films deposited by atomic vapor deposition," Appl. Phys. Lett., 101[11] 112903 (2012).

40. S.-1. Ahn, Y. Noguchi, M. Miyayama, andT. Kudo, "Structural and electrical characterization of $\mathrm{Bi}_{5} \mathrm{Ti}_{3} \mathrm{Fe}_{1-\mathrm{x}} \mathrm{Mn}_{\mathrm{x}} \mathrm{O}_{15}$ system," Materials Research Bulletin, 35[6] 825-34 (2000).

41. L. Keeney, C. Groh, S. Kulkarni, S. Roy, M. E. Pemble, andR. W. Whatmore, "Room temperature electromechanical and magnetic investigations of ferroelectric Aurivillius 
phase $\mathrm{Bi}_{5} \mathrm{Ti}_{3}\left(\mathrm{Fe}_{\mathrm{x}} \mathrm{Mn}_{1-\mathrm{x}}\right) \mathrm{O}_{15}(\mathrm{x}=1$ and 0.7$)$ chemical solution deposited thin films," $J$. Appl. Phys., 112[2] 024101 (2012).

42. N. A. Lomanova, M. I. Morozov, V. L. Ugolkov, andV. V. Gusarov, "Properties of aurivillius phases in the Bi4Ti3O12-BiFeO3 system," Inorg. Mat., 42[2] 189-95 (2006).

43. D. Y. Suarez, I. M. Reaney, andW. E. Lee, "Relation between tolerance factor and Tc in Aurivillius compunds," J. Mater. Res., 16[11] 3139-49 (2001).

44. A. M. Glazer, "The Classification of Titled Octahedra in Perovskites," Acta Cryst., B28 3384-92 (1972).

45. C. H. Hervoches, A. Snedden, R. Riggs, S. H. Kilcoyne, P. Manuel, andP. Lightfoot, "Structural Behavior of the Four-Layered Aurivillius-Phase Ferroelectrics $\mathrm{SrBi}_{4} \mathrm{Ti}_{4} \mathrm{O}_{14}$ and $\mathrm{Bi}_{5} \mathrm{Ti}_{3} \mathrm{FeO}_{15}, "$ J. Solid State Chem., 164 280-91 (2002).

46. A. Sanson and R. W. Whatmore, "Properties of $\mathrm{Bi}_{4} \mathrm{Ti}_{3} \mathrm{O}_{12}-\left(\mathrm{Na}_{1 / 2} \mathrm{Bi}_{1 / 2}\right) \mathrm{TiO}_{3}$ Piezoelectric Ceramics," Jpn. J. Appl. Phys. Part 1, 41[11B] 7127-30 (2002).

47. A. Sanson and R. W. Whatmore, "Phase Diagram of the $\mathrm{Bi}_{4} \mathrm{Ti}_{3} \mathrm{O}_{12}-\mathrm{BaTiO}_{3}-$ $\left(\mathrm{Na}_{1 / 2} \mathrm{Bi}_{1 / 2}\right) \mathrm{TiO}_{3}$ System," J. Am. Ceram. Soc., 88[11] 3147-53 (2005).

48. P. Boullay, G. Trolliard, D. Mercurio, J. M. Perez-Mato, andL. Elcoro, "Toward a Unified Approach to the Crystal Chemistry of Aurivillius-Type Compounds.: I. The Structural Model," Journal of Solid State Chemistry, 164[2] 252-60 (2002).

49. X. Y. Mao, W. Wang, andX. B. Chen, "Electrical and magnetic properties of Bi5FeTi3O15 compound prepared by inserting BiFeO3 into Bi4Ti3O12," Solid State Commun., 147[5-6] 186-89 (2008).

50. A. Srinivas, M. M. Kumar, S. V. Suryanarayana, andT. Bhimasankaram, "Investigation of dielectric and magnetic nature of $\mathrm{Bi}_{7} \mathrm{Fe}_{3} \mathrm{Ti}_{3} \mathrm{O}_{21}$," Materials Research Bulletin, 34[6] 989-96 (1999). 
51. A. Srinivas, D.-W. Kim, K. S. Hong, andS. V. Suryanarayana, "Study of magnetic and magnetoelectric measurements in bismuth iron titanate ceramic $-\mathrm{Bi}_{8} \mathrm{Fe}_{4} \mathrm{Ti}_{3} \mathrm{O}_{24}$," Materials Research Bulletin, 39[1] 55-61 (2004).

52. M. A. Zurbuchen, R. S. Freitas, M. J. Wilson, P. Schiffer, M. Roeckerath, J. Schubert, M. D. Biegalski, G. H. Mehta, D. J. Comstock, J. H. Lee, Y. Jia, andD. G. Schlom, "Synthesis and characterization of an $\mathrm{n}=6$ Aurivillius phase incorporating magnetically active manganese, $\mathrm{Bi}_{7}(\mathrm{Mn}, \mathrm{Ti})_{6} \mathrm{O}_{21}, "$ Appl. Phys. Lett., 91[3] 033113 (2007).

53. X. Mao, W. Wang, X. Chen, andY. Lu, "Multiferroic properties of layer-structured $\mathrm{Bi}_{5} \mathrm{Fe}_{0.5} \mathrm{Co}_{0.5} \mathrm{Ti}_{3} \mathrm{O}_{15}$ ceramics," Appl. Phys. Lett., 95[8] 082901 (2009).

54. L. Keeney, S. Kulkarni, N. Deepak, M. Schmidt, N. Petkov, P. F. Zhang, S. Cavill, S. Roy, M. E. Pemble, andR. W. Whatmore, "Room temperature ferroelectric and magnetic investigations and detailed phase analysis of Aurivillius phase $\mathrm{Bi}_{5} \mathrm{Ti}_{3} \mathrm{Fe}_{0.7} \mathrm{Co}_{0.3} \mathrm{O}_{15}$ thin films," J. Appl. Phys., 112[5] 052010 (2012).

55. M. Palizdar, T. P. Comyn, M. B. Ward, A. P. Brown, J. P. Harrington, S. Kulkarni, L. Keeney, S. Roy, M. E. Pemble, R. W. Whatmore, C. Quinne, S. H. Kilcoyne, andA. J. Bell, "Cystallographic and Magnetic Identification of Secondary Phase in Oriented $\mathrm{Bi}_{5} \mathrm{Fe}_{0.5} \mathrm{Co}_{0.5} \mathrm{Ti}_{3} \mathrm{O}_{15}$ Ceramics," J. Appl. Phys., 112[7] 073919 (2012).

56. F. Z. Huang, X. M. Lu, T. T. Xu, Y. Y. Liu, W. N. Su, Y. M. Jin, Y. Kan, andJ. S. Zhu, "Multiferroic properties of Co and Nd co-substituted $\mathrm{Bi}_{5} \mathrm{Ti}_{3} \mathrm{FeO}_{15}$ thin films," Thin Solid Films, 520[21] 6489-92 (2012).

57. J. Yang, W. Tong, Z. Liu, X. B. Zhu, J. M. Dai, W. H. Song, Z. R. Yang, andY. P. Sun, "Structural, magnetic, and EPR studies of the Aurivillius phase $\mathrm{Bi}_{6} \mathrm{Fe}_{2} \mathrm{Ti}_{3} \mathrm{O}_{18}$ and Bi $_{6}$ FeCrTi $_{3} \mathrm{O}_{18}, "$ Phys. Rev. B, 86[10] 104410 (2012). 
58. J. Yang, L. H. Yin, Z. Liu, X. B. Zhu, W. H. Song, J. M. Dai, Z. R. Yang, andY. P. Sun, "Magnetic and dielectric properties of Aurivillius phase $\mathrm{Bi}_{6} \mathrm{Fe}_{2} \mathrm{Ti}_{3} \mathrm{O}_{18}$ and the doped compounds," Appl. Phs. Lett., 101[1] 012402 (2012).

59. N. V. Prasad and G. S. Kumar, "Magnetic and magnetoelectric measurements on rareearth-substituted five-layered $\mathrm{Bi}_{6} \mathrm{Fe}_{2} \mathrm{Ti}_{3} \mathrm{O}_{12}$ compound," J. Magn. Magn. Mater., 213 349-56 (2000).

60. Z. Liu, J. Yang, X. W. Tang, L. H. Yin, X. B. Zhu, J. M. Dai, andY. P. Sun, "Multiferroic properties of Aurivillius phase $\mathrm{Bi}_{6} \mathrm{Fe}_{2-\mathrm{x}} \mathrm{Co}_{\mathrm{x}} \mathrm{Ti}_{3} \mathrm{O}_{18}$ thin films prepared by a chemical solution deposition route," Appl. Phs. Lett., 101[12] (2012).

61. F. K. Lotgering, "Topotactical reactions with ferrimagnetic oxides having hexagonal crystal structures-I," J. Inorg. Nucl. Chem., 9[2] 113-23 (1959).

62. "Crystallographica, v1.60d, (c) Oxford Cryosystems Ltd," v1.60d (1995-2007).

63. P. Kuisma-Kursula and J. RÄIsÄNen, "SCANNING ELECTRON MICROSCOPYENERGY DISPERSIVE SPECTROMETRY AND PROTON INDUCED X-RAY EMISSION ANALYSES OF MEDIEVAL GLASS FROM KOROINEN (FINLAND)*," Archaeometry, 41[1] 71-79 (1999).

64. B. J. Rodriguez, C. Callahan, S. V. Kalinin, andR. Proksch, "Dual-frequency resonancetracking atomic force microscopy," Nanotechnology, 18[47] 475504 (2007).

65. S. Jesse, A. P. Baddorf, andS. V. Kalinin, "Switching spectroscopy piezoresponse force microscopy of ferroelectric materials," Appl. Phys. Lett., 88[6] 062908 (2006).

66. S. Jesse, H. N. Lee, andS. V. Kalinin, "Quantitative mapping of switching behavior in piezoresponse force microscopy," pp. 073702. in, Vol. 77. AIP, 2006.

67. A. Gruverman, O. Auciello, R. Ramesh, andH. Tokumoto, "Scanning force microscopy of domain structure in ferroelectric thin films: imaging and control," Nanotechnology, 8[3A] A38-A43 (1997). 
68. S. V. Kalinin, B. J. Rodriguez, S. Jesse, E. Karapetian, B. Mirman, E. A. Eliseev, andA. N. Morozovska, "Nanoscale Electromechanics of Ferroelectric and Biological Systems: A New Dimension in Scanning Probe Microscopy," Ann. Rev. of Mater. Res., 37[1] 189-238 (2007).

69. N. Balke, I. Bdikin, S. V. Kalinin, andA. L. Kholkin, "Electromechanical Imaging and Spectroscopy of Ferroelectric and Piezoelectric Materials: State of the Art and Prospects for the Future," J. Am. Ceram. Soc., 92[8] 1629-47 (2009).

70. S. V. Kalinin, B. J. Rodriguez, S. Jesse, P. Maksymovych, K. Seal, M. Nikiforov, A. P. Baddorf, A. L. Kholkin, andR. Proksch, "Local bias-induced phase transitions," Materials Today, 11[11] 16-27 (2008).

71. E. Soergel, "Piezoresponse Force Microscopy (PFM)," J. Phys. D: Appl. Phys., 44 464003 (2011).

72. H. Chang, S. V. Kalinin, S. Yang, P. Yu, S. Bhattacharya, P. P. Wu, N. Balke, S. Jesse, L. Q. Chen, R. Ramesh, S. J. Pennycook, andA. Y. Borisevich, "Watching domains grow: In-situ studies of polarization switching by combined scanning probe and scanning transmission electron microscopy," Journal of Applied Physics, 110[5] 052014 (2011).

73. S. V. Kalinin, A. Gruverman, andD. A. Bonnell, "Quantitative analysis of nanoscale switching in $\mathrm{SrBi}_{2} \mathrm{Ta}_{2} \mathrm{O}_{9}$ thin films by piezoresponse force microscopy," Appl. Phys. Lett., 85[5] 795-97 (2004).

74. "VFM2 ${ }^{\text {тм }}$ Variable Field Module for Magnetic AFM

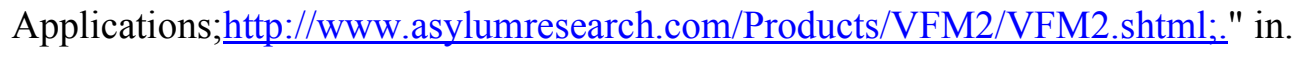

75. A. Barone, "Principles and Applications of Superconducting Quantum Interference Devices ". World Scientific, (1992). 
76. P. K. Foster and J. E. Welch, "METAL-OXIDE SOLID SOLUTIONS. PART 1.LATTICE-CONSTANT AND PHASE RELATIONSHIPS IN FERROUS OXIDE (WUSTITE) AND IN SOLID SOLUTIONS OF FERROUS OXIDE AND MANGANOUS OXIDE," Trans. Faraday Soc., 521626 (1956).

77. D. A. Hope, A. K. Cheetham, andG. J. Long, "A Neutron Diffraction, Magnetic Susceptibility, and Mossbauer-Effect Study of the $\left(\mathrm{Mn}_{\mathrm{x}} \mathrm{Fe}_{1-\mathrm{x}}\right)_{\mathrm{y}} \mathrm{O}$ Solid Solutions," Inorg. Chem., 21 2804-09 (1982).

78. "Crystal Studio Version 12 Quantum edition."

79. J. Reyes-Gasga, A. Gómez-Rodríguez, X. Gao, andM. José-Yacamán, "On the interpretation of the forbidden spots observed in the electron diffraction patterns of flat Au triangular nanoparticles," Ultramicroscopy, 108[9] 929-36 (2008).

80. M. Retuerto, M. R. Li, Y. B. Go, A. Ignatov, M. Croft, K. V. Ramanujachary, R. H. Herber, I. Nowik, J. P. Hodges, W. Dachraoui, J. Hadermann, andM. Greenblatt, "High magnetic ordering temperature in the perovskites $\mathrm{Sr}_{4-\mathrm{x}} \mathrm{La}_{\mathrm{x}} \mathrm{Fe}_{3} \mathrm{ReO}_{12}(\mathrm{x}=0.0,1.0$, 2.0)," J. Solid State Chem., 194[0] 48-58 (2012).

81. J. Mizusaki, N. Mori, H. Takai, Y. Yonemura, H. Minamiue, H. Tagawa, M. Dokiya, H. Inaba, K. Naraya, T. Sasamoto, andT. Hashimoto, "Oxygen nonstoichiometry and defect equilibrium in the perovskite-type oxides La1-xSrxMnO3+d," Solid State Ionics, 129[1-4] 163-77 (2000).

82. J. Varghese, S. Barth, L. Keeney, R. W. Whatmore, andJ. D. Holmes, "Nanoscale Ferroelectric and Piezoelectric Properties of Sb2S3 Nanowire Arrays," Nano Lett., 12[2] 868-72 (2012).

83. S. V. Kalinin, Z.-G. Ye, andA. L. Kholkin, "Preface to Special Topic: Piezoresponse Force Microscopy and Nanoscale Phenomena in Polar Materials," J. Appl. Phys., 112[5] 051901 (2012). 
84. S. V. Kalinin, N. Setter, andA. L. Kholkin, "Electromechanics on the Nanometer Scale: Emerging Phenomena, Devices, and Applications," Mrs Bulletin, 34[09] 634-42 (2009).

85. T. Watanabe and H. Funakubo, "Controlled crystal growth of layered-perovskite thin films as an approach to study their basic properties," J. Appl. Phys., 100[5] 051602 (2006).

86. J. Yang, L. H. Yin, Z. Liu, X. B. Zhu, W. H. Song, J. M. Dai, Z. R. Yang, andY. P. Sun, "Magnetic and dielectric properties of Aurivillius phase $\mathrm{Bi}_{6} \mathrm{Fe}_{2} \mathrm{Ti}_{3} \mathrm{O}_{18}$ and the doped compounds," Appl. Phys. Lett., 101[1] 012402 (2012).

87. J. Lu, L. J. Qiao, X. Q. Ma, andW. Y. Chu, "Magnetodielectric effect of $\mathrm{Bi}_{6} \mathrm{Fe}_{2} \mathrm{Ti}_{3} \mathrm{O}_{18}$ film under an ultra-low magnetic field," J. Phys.: Condens. Matter., 18 4801-07 (2006).

88. A. Srinivas, S. V. Suryanarayana, G. S. Kumar, andM. M. Kumar, "Magnetoelectric measurements on $\mathrm{Bi}_{5} \mathrm{FeTi}_{3} \mathrm{O}_{15}$ and $\mathrm{Bi}_{6} \mathrm{Fe}_{2} \mathrm{Ti}_{3} \mathrm{O}_{18}$," J. Phys.: Condens. Matter., 11 3335-40 (1999).

89. A. Gruverman and Y. Ikeda, "Characterization and Control of Domain Structure in SrBi2Ta2O9 Thin Films by Scanning Force Microscopy," Jpn. J. Appl. Phys., Part 2, 37[8A] L939-L41 (1998).

90. "calculated using Image J 1.44p."

91. S. Chikazumi and S. H. Charap, "Physics of Magnetism." Wiley: New York, (1964).

92. R. S. Tebble and D. J. Craik, "Magnetic Materials." Wiley: New York, (1969).

93. G. F. Goya, T. S. Berquo, F. C. Fonseca, andM. P. Morales, "Static and dynamic magnetic properties of spherical magnetite nanoparticles," J. Appl. Phys., 94[5] 352028 (2003). 
94. N. N. Guan, Y. T. Wang, D. J. Sun, andJ. Xu, "A simple one-pot synthesis of singlecrystalline magnetite hollow spheres from a single iron precursor," Nanotechnology, 20[10] (2009).

95. J. B. Goodenough, "Theory of the Role of Covalence in the Perovsjite-type Manganites [La, M(II)] $\mathrm{MnO}_{3}$," Phys. Rev., 100[2] 564-73 (1955).

96. "Mercury 3.0 (Build RC5)," (2001-2011). 


\section{List of Figures:}

Fig. 1. (a) XRD patterns from B7TFO and B6TFMO thin films, (b) HR-TEM image and (c) electron diffraction pattern of B6TFMO. Note that $\log$ (intensity) vs $2 \theta$ is used here in the XRD pattern to make-clear the weakest peaks relative to the strongest.

Fig. 2. Representative (a) AFM image of B7TFO thin films and (b) HR-SEM image of B6TFMO thin films on $c$-plane sapphire.

Fig. 3. Compositional maps produced by extended period ( 72 hour) data collections from a $1600 \mu \mathrm{m}^{2}$ sample area, followed by subtraction of the Bi L $\alpha$ from the $\mathrm{Fe} \mathrm{K} \alpha$ and $\mathrm{Mn} \mathrm{K} \alpha$ signals. These show a) regions of excess Fe in B7TFO b) regions of excess Fe in B6TFMO and c) regions of excess $\mathrm{Mn}$ in B6TFMO. Note the one-to-one correspondence between the small, bright (numbered) regions showing Fe in b) and Mn excesses in $\mathbf{c}$ ). Note also the larger pale areas in b) corresponding to areas where the Fe content slightly exceeds the surrounding grains. d) Cross-sectional HR-TEM image and e) diffraction pattern taken from a single $\mathrm{Mn}_{0.53} \mathrm{Fe}_{0.47} \mathrm{O}$ inclusion. f) Cross-sectional HR-TEM image and g) diffraction pattern taken from a single higher-Fe content Aurivillius grain within B6TFMO.

Fig. 4. Dark field TEM analysis of a single $\mathrm{Mn}_{0.53} \mathrm{Fe}_{0.47} \mathrm{O}$ inclusion. These show a) SAED pattern b) - i) dark field images taken from the spots indicated in a), crystal direction indicated in each image. The sapphire substrate is on the left side of the images. 
Fig. 5. a) Plot of the normalized B-site composition of Fe and Mn vs that for Ti, as determined by cross-section HR-STEM-EDX from 55 Aurivillius grains within the B6TFMO sample. b) Plot of the total B-site Mn composition and B-site $\mathrm{Mn}^{4+}$ composition, calculated to maintain charge balance, vs the B-site Fe composition.

Fig. 6. Representative (a) topography, (b) lateral single frequency PFM amplitude, (c) lateral single frequency PFM phase, (d) vertical single frequency PFM amplitude and (e) vertical single frequency PFM phase images of B7TFO thin films on $c$-plane sapphire and (f) topography, (g) lateral single frequency PFM amplitude, (h) lateral single frequency PFM phase, (i) vertical single frequency PFM amplitude and (j) vertical single frequency PFM phase images of B6TFMO thin films on $c$-plane sapphire.

Fig. 7. Representative (a) topography, (b) vertical DART-PFM amplitude and (c) vertical DART-PFM phase images of B6TFMO thin films on $c$-plane sapphire.

Fig. 8. Vertical DART-PFM switching spectroscopy (a) phase and (b) piezoresponse loops of B7TFO and (c) phase and (d) piezoresponse loops of B6TFMO thin films in the absence of an applied DC bias. Images of B6TFMO on c-plane sapphire: (e) out-of-plane PFM phase and (f) out-of-plane PFM amplitude after PFM lithography with an applied electric field of 70 $\mathrm{V}$.

Fig. 9. (a) $M$ vs. $H$ and (b) M vs. T measurements (ZFC \& FC) for B6TFMO on sapphire. Inset image in (a) shows SEM image of sample with $2.44 \%$ pores (red arrows) (c) Magnetic parameters for B6TFMO thin film on sapphire. (d) M Vs T measurement for B7TFO. 
Fig. 10. The magnetic properties of B 7 TFO phase were investigated. Figure (a) shows the magnetic hysteresis measured at $2 \mathrm{~K}$ and the inset shows hysteresis after direct subtraction of the diamagnetic substrate contribution. Figure (b) shows the zoomed hysteresis loop of B6TFMO measured at different temperatures.

Fig. 11. Representative images of B6TFMO thin films: (a) topography, (c) lateral PFM amplitude and (e) lateral PFM phase under $0 \mathrm{mT}$ (-1.9 Oe) H field and (b) topography, (d) lateral PFM and (f) lateral PFM phase under +250 mT (+2501 Oe) H field.

Fig. 12. Representative images of B6TFMO thin films: (a) topography, (b) vertical PFM amplitude and (c) vertical PFM phase under $0 \mathrm{mT}$ (-0.9 Oe) H field,(d) topography, (e) vertical PFM amplitude and (f) vertical PFM phase under $+250 \mathrm{mT}(+2501 \mathrm{Oe}) \mathrm{H}$ field and (g) topography, (h) vertical PFM amplitude and (i) vertical PFM phase under -250 mT (-2501 Oe) $\mathrm{H}$ field. 


\section{List of Figures - Sidebars:}

Fig. A.1. The relationship between multiferroic and magnetoelectric materials. (Redrawn from $\left.^{23}\right)$.

Fig. B.1. Projection (approximately down (101)) of half-unit cells of (a) $\mathrm{Bi}_{7} \mathrm{Ti}_{3} \mathrm{Fe}_{3} \mathrm{O}_{21}$ and (b) $\mathrm{Bi}_{6} \mathrm{Ti}_{3} \mathrm{Fe}_{1.6} \mathrm{Mn}_{0.6} \mathrm{O}_{18}$ displaying the in-plane lattice directions (100) (dashed arrow) and (110) (yellow plane). Drawn using Crystallographica v1.60d ${ }^{62}$ and Mercury 3.0 Crystal Structure Visualization software ${ }^{96}$.

Fig. C.1. (a) Schematic representation of vertical PFM operation and (b) triangle step bias waveform applied to the sample during switching-spectroscopy PFM hysteresis loop acquisition.

Fig. D.1. Change in susceptibility $(\chi)$ versus temperature $(T)$ for different types of magnetic materials

Fig. D.2. Equivalent circuit of the SQUID magnetometer

Fig. D.3. Typical Zero Field Cooled (ZFC) - Field Cooled (FC) - Remanence curve (a) and magnetic hysteresis (MH) loop (b) measured in SQUID magnetometer 


\section{List of Tables - Main Document}

Table 1. Performed Volume Scans

\begin{tabular}{|c|c|c|c|c|c|}
\hline Method & $k$ & $\begin{array}{c}\text { Volume } V_{k} \\
{\left[\mu \mathrm{m}^{3}\right]}\end{array}$ & $\begin{array}{c}\text { smallest diameter } d_{k} \\
{[\mathrm{~nm}]}\end{array}$ & $\phi_{k}$ & $M_{r, k}$ \\
& & & & & \\
\hline Surface EDX & 1 & 2000 & $1000\left(\mathrm{~d}_{0}=5 \mu \mathrm{m}\right)$ & $0.010 \%$ & 2.1 \\
\hline Surface EDX & 2 & 450 & 350 & $0.006 \%$ & 1.1 \\
\hline Surface EDX & 3 & 120 & 100 & $0.005 \%$ & 1.0 \\
\hline Surface EDX & 4 & 28 & 60 & $0.001 \%$ & 0.2 \\
\hline Surface EDX & 5 & 7.2 & 20 & $0.008 \%$ & 1.7 \\
\hline TEM & 6 & 0.08 & 2 & $0.028 \%$ & 2.8 \\
\hline
\end{tabular}

30

31

32

33

34

35

36

37

38

39

40

41

42

43

44

45

46

47

48

49

50

51

52

53

54

55

56 


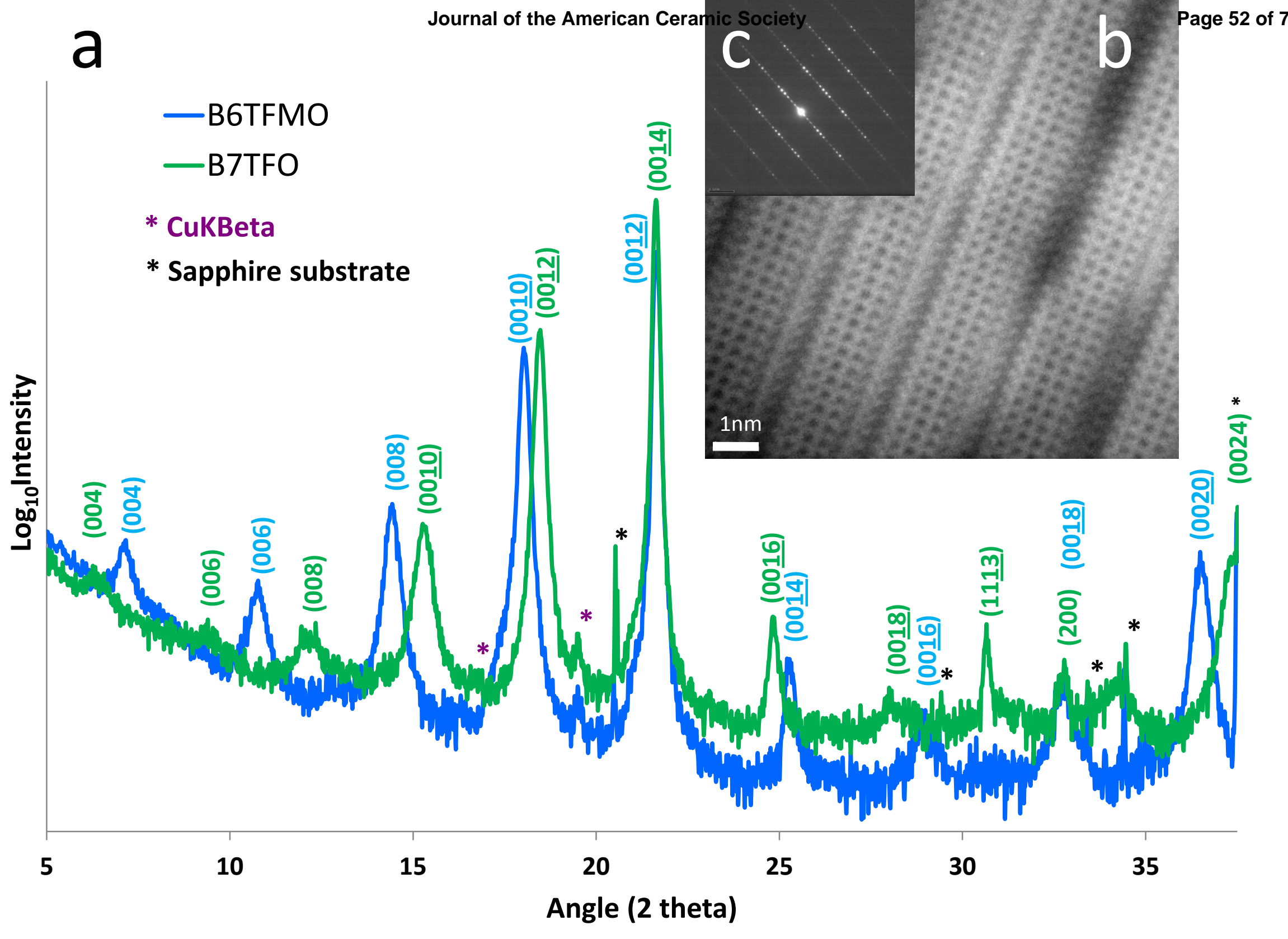

Journal of the American Ceramic Society 

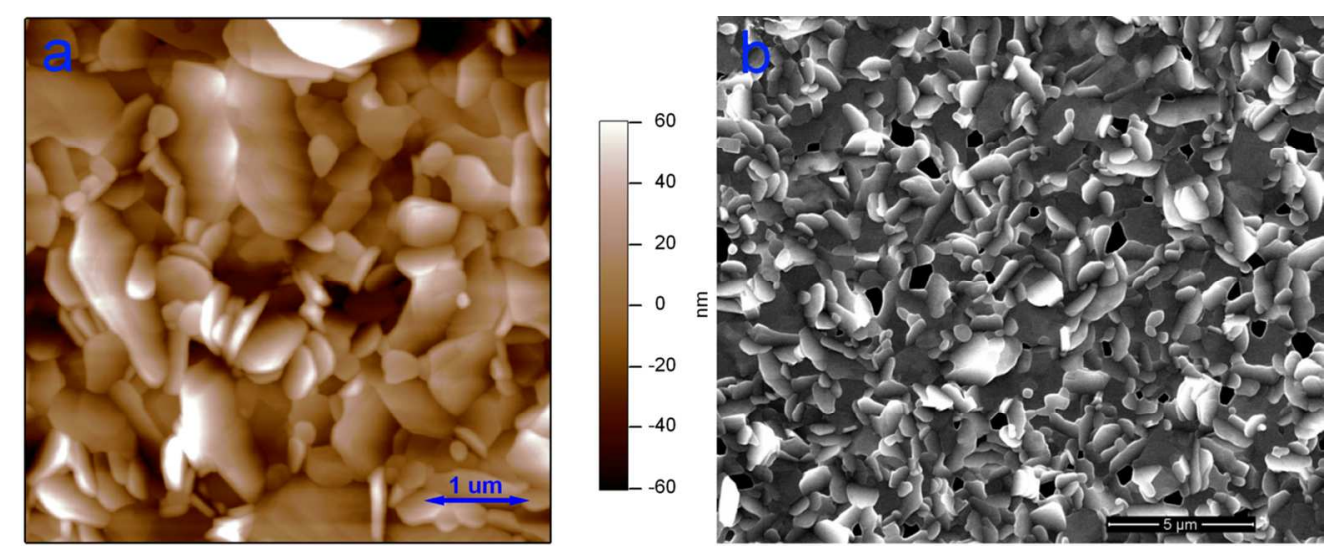

Fig. 2. Representative (a) AFM image of B7TFO thin films and (b) HR-SEM image of B6TFMO thin films on cplane sapphire. $127 \times 51 \mathrm{~mm}(300 \times 300 \mathrm{DPI})$

23
24
25
26
27
28
29
30
31
32
33
34
35
36
37
38
39
40
41
42
43
44
45
46
47
48
49
50
51
52
53
54
55
56
57
58
59
60



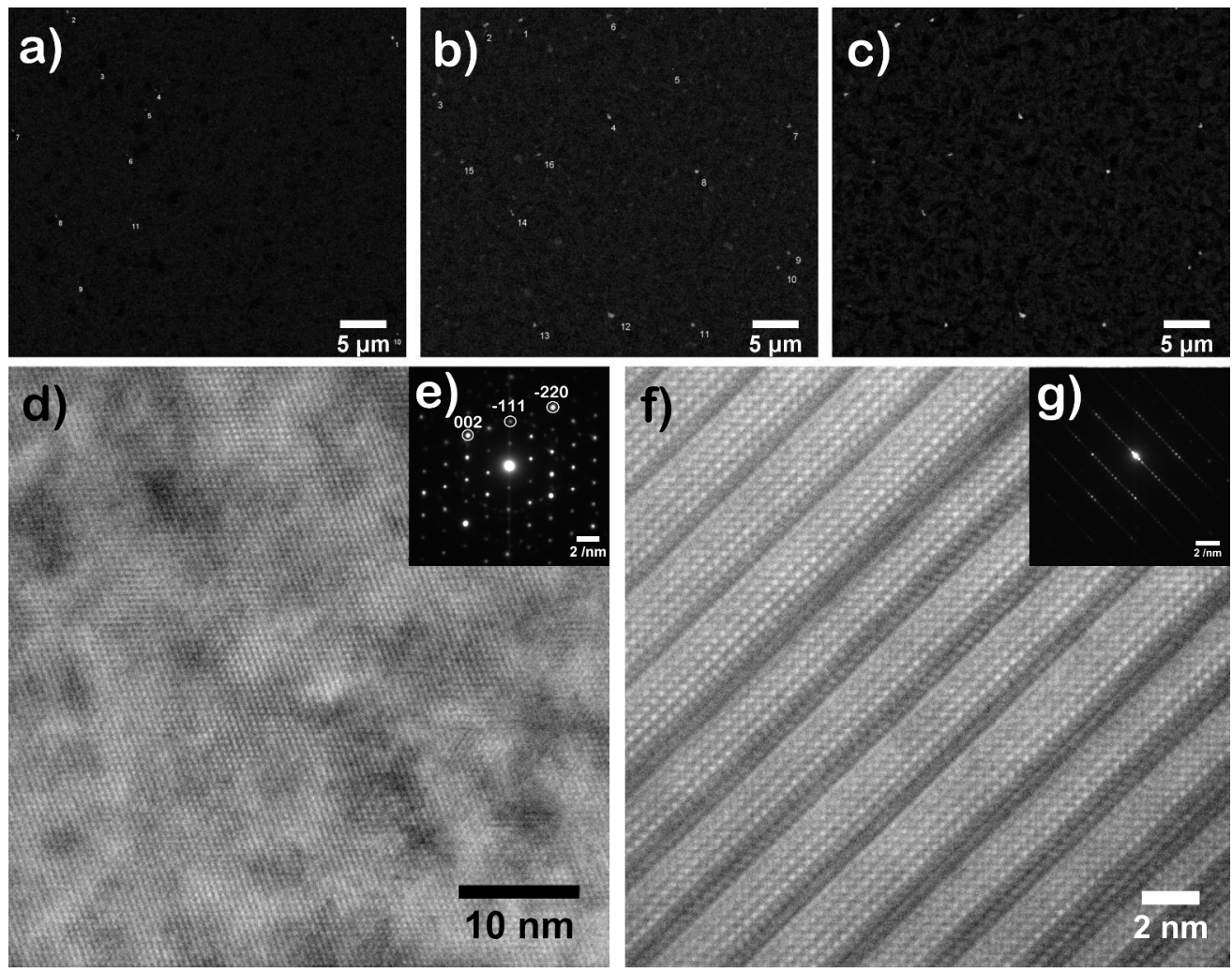

Fig. 3. Compositional maps produced by extended period ( 72 hour) data collections from a $1600 \mu m^{2}$ sample area, followed by subtraction of the Bi La from the $\mathrm{Fe} \mathrm{Ka}$ and Mn Ka signals. These show a) regions of excess Fe in B7TFO b) regions of excess Fe in B6TFMO and c) regions of excess Mn in B6TFMO. Note the one-to-one correspondence between the small, bright (numbered) regions showing $\mathrm{Fe}$ in $\mathrm{b}$ ) and $\mathrm{Mn}$ excesses in c). Note also the larger pale areas in b) corresponding to areas where the Fe content slightly exceeds the surrounding grains. d) Cross-sectional HR-TEM image and e) diffraction pattern taken from a single $\mathrm{Mn}_{0.53} \mathrm{Fe}_{0.47} \mathrm{O}$ inclusion. f) Cross-sectional HR-TEM image and g) diffraction pattern taken from a single higher-Fe content Aurivillius grain within B6TFMO. $266 \times 209 \mathrm{~mm}(300 \times 300$ DPI) 


\section{Page 55 of 70}

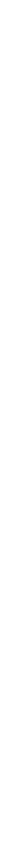



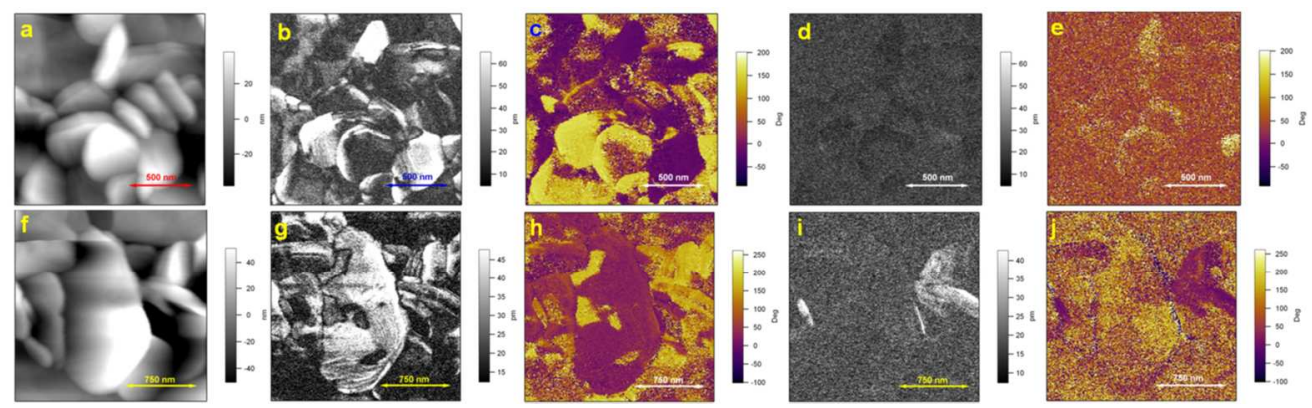

Fig. 6. Representative (a) topography, (b) lateral single frequency PFM amplitude, (c) lateral single frequency PFM phase, (d) vertical single frequency PFM amplitude and (e) vertical single frequency PFM phase images of B7TFO thin films on c-plane sapphire and (f) topography, $(\mathrm{g}$ ) lateral single frequency PFM amplitude, (h) lateral single frequency PFM phase, (i) vertical single frequency PFM amplitude and (j) vertical single frequency PFM phase images of B6TFMO thin films on c-plane sapphire.

\section{$127 \times 38 \mathrm{~mm}(300 \times 300$ DPI $)$}



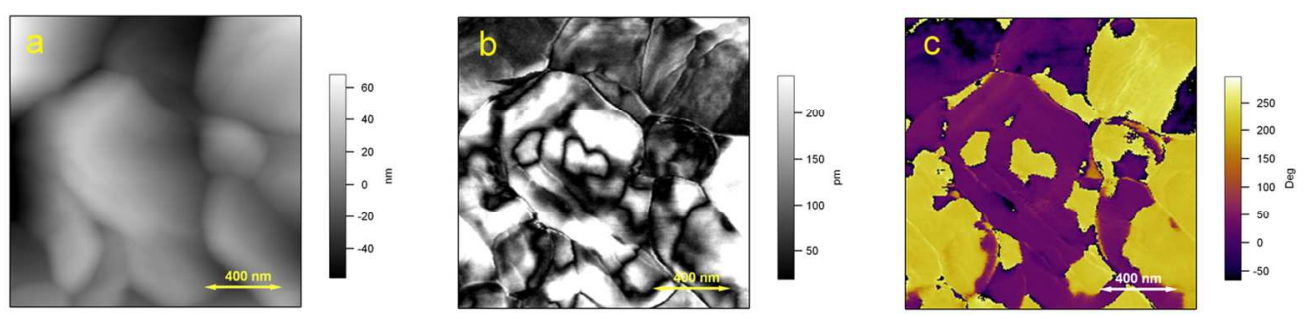

Fig. 7. Representative (a) topography, (b) lateral single frequency PFM amplitude, (c) lateral single frequency PFM phase, (d) vertical single frequency PFM amplitude and (e) vertical single frequency PFM phase images of B7TFO thin films on c-plane sapphire and (f) topography, $(\mathrm{g}$ ) lateral single frequency PFM amplitude, (h) lateral single frequency PFM phase, (i) vertical single frequency PFM amplitude and (j) vertical single frequency PFM phase images of B6TFMO thin films on c-plane sapphire. $127 \times 29 \mathrm{~mm}(300 \times 300 \mathrm{DPI})$ 


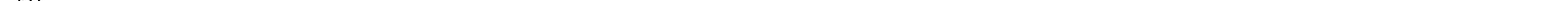



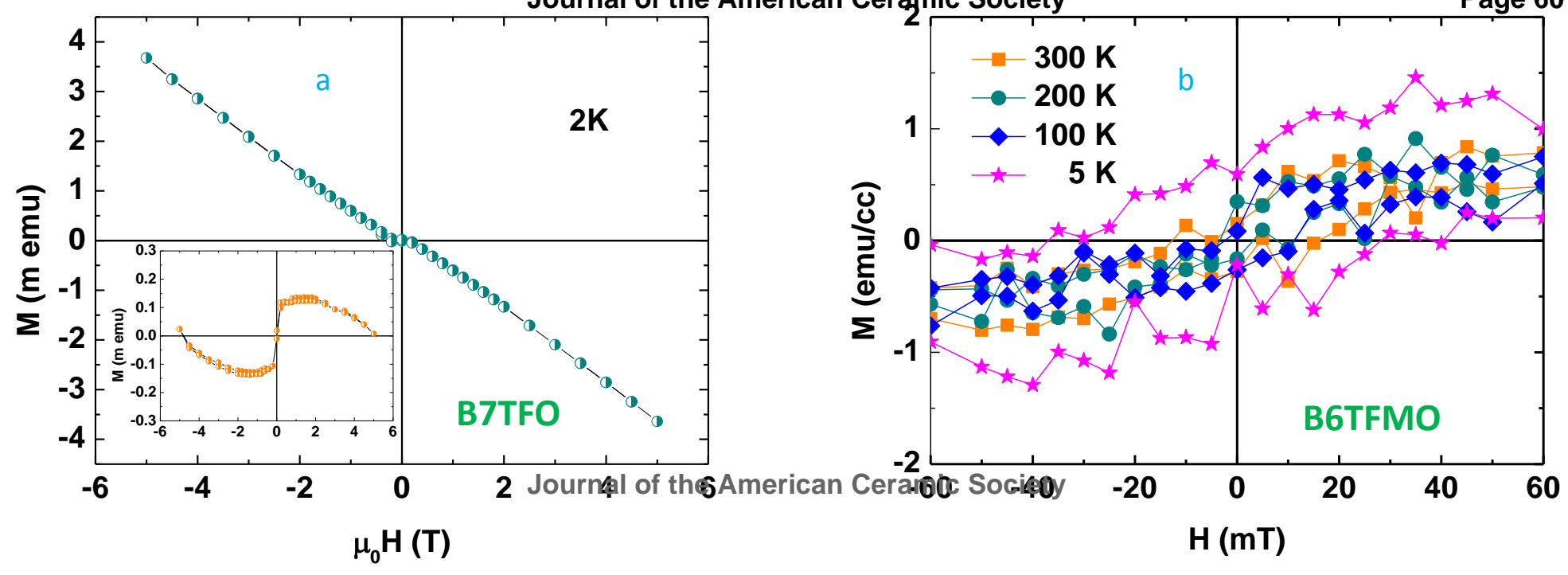


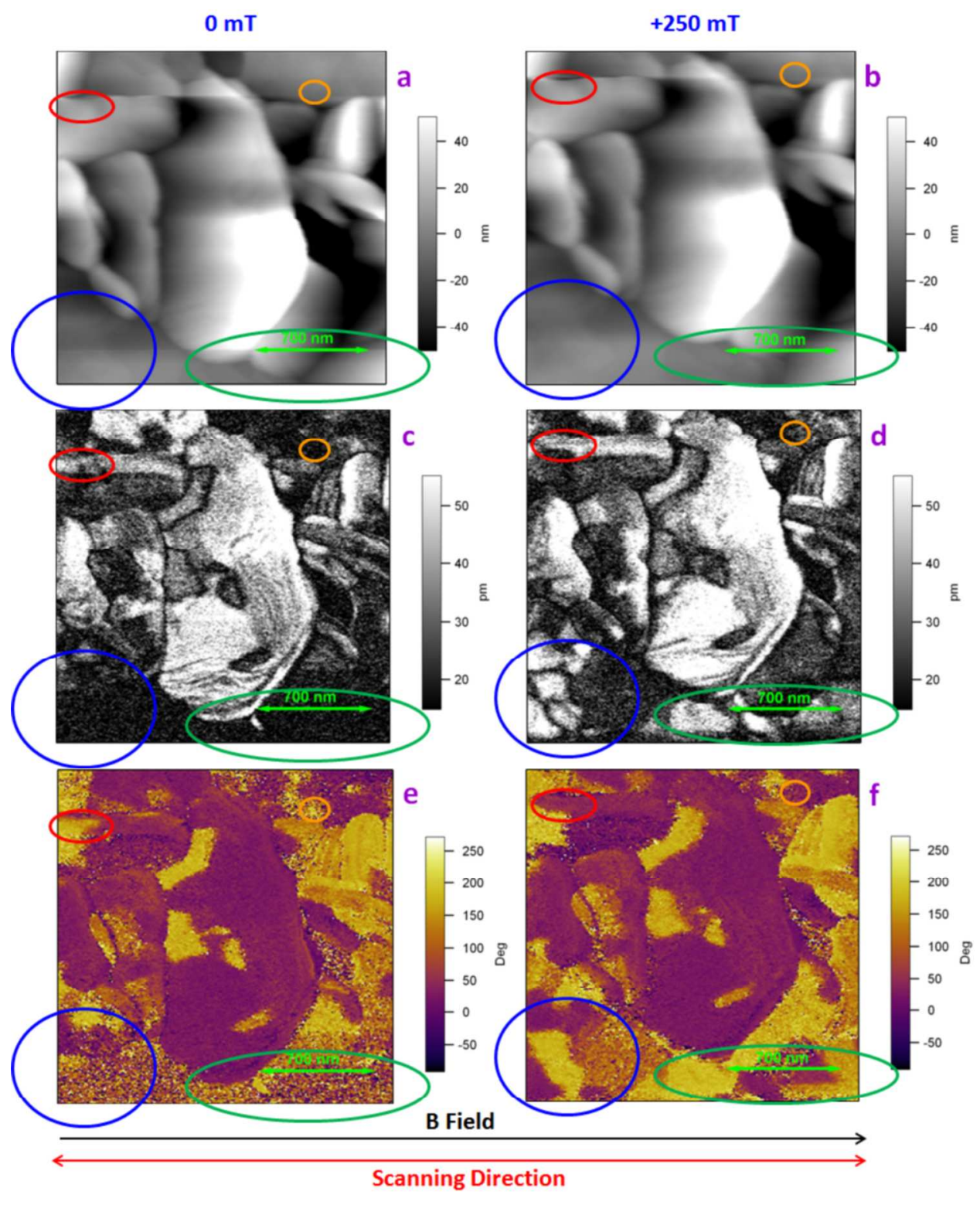

Fig. 11. Representative images of B6TFMO thin films: (a) topography, (c) lateral PFM amplitude and (e) lateral PFM phase under $0 \mathrm{mT}(-1.9 \mathrm{Oe}) \mathrm{H}$ field and (b) topography, (d) lateral PFM and (f) lateral PFM phase under $+250 \mathrm{mT}(+2501 \mathrm{Oe}) \mathrm{H}$ field. $238 \times 297 \mathrm{~mm}(300 \times 300 \mathrm{DPI})$ 

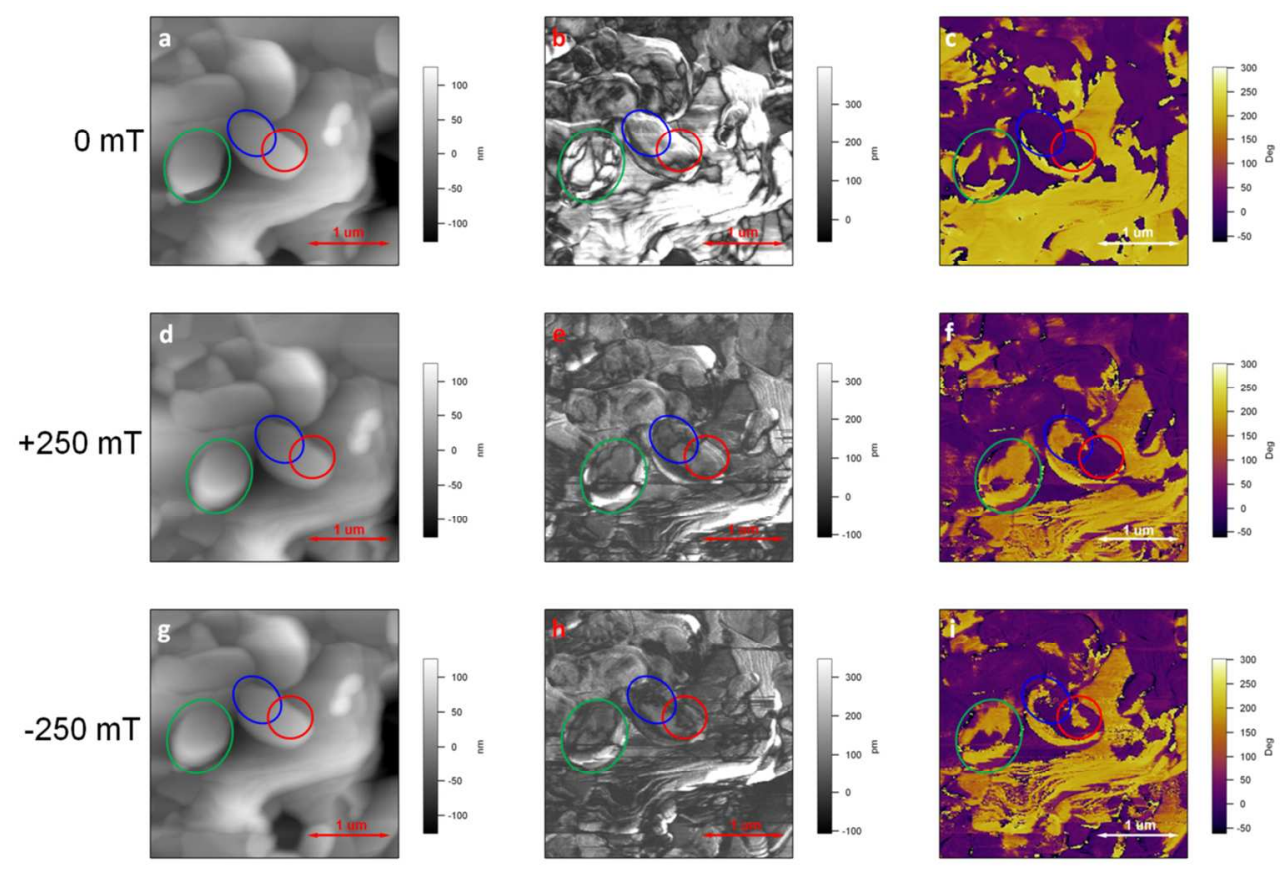

B Field

Scanning Direction

Fig. 12. Representative images of B6TFMO thin films: (a) topography, (b) vertical PFM amplitude and (c) vertical PFM phase under $0 \mathrm{mT}(-0.9 \mathrm{Oe}) \mathrm{H}$ field,(d) topography, (e) vertical PFM amplitude and (f) vertical PFM phase under $+250 \mathrm{mT}(+2501 \mathrm{Oe}) \mathrm{H}$ field and $(\mathrm{g})$ topography, $(\mathrm{h})$ vertical PFM amplitude and (i) vertical PFM phase under $-250 \mathrm{mT}(-2501 \mathrm{Oe}) \mathrm{H}$ field. $127 \times 99 \mathrm{~mm}(300 \times 300 \mathrm{DPI})$ 


\section{Page 63 of 70}

Journal of the American Ceramic Society

1
2
3
4
5
6
7
8
9

Ferromagnetic

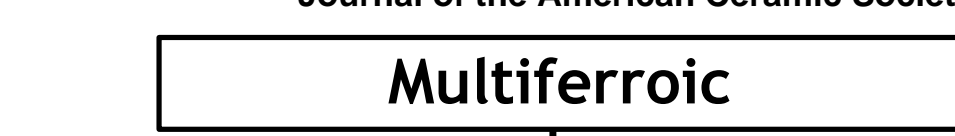

Memory \& Sensor Applications

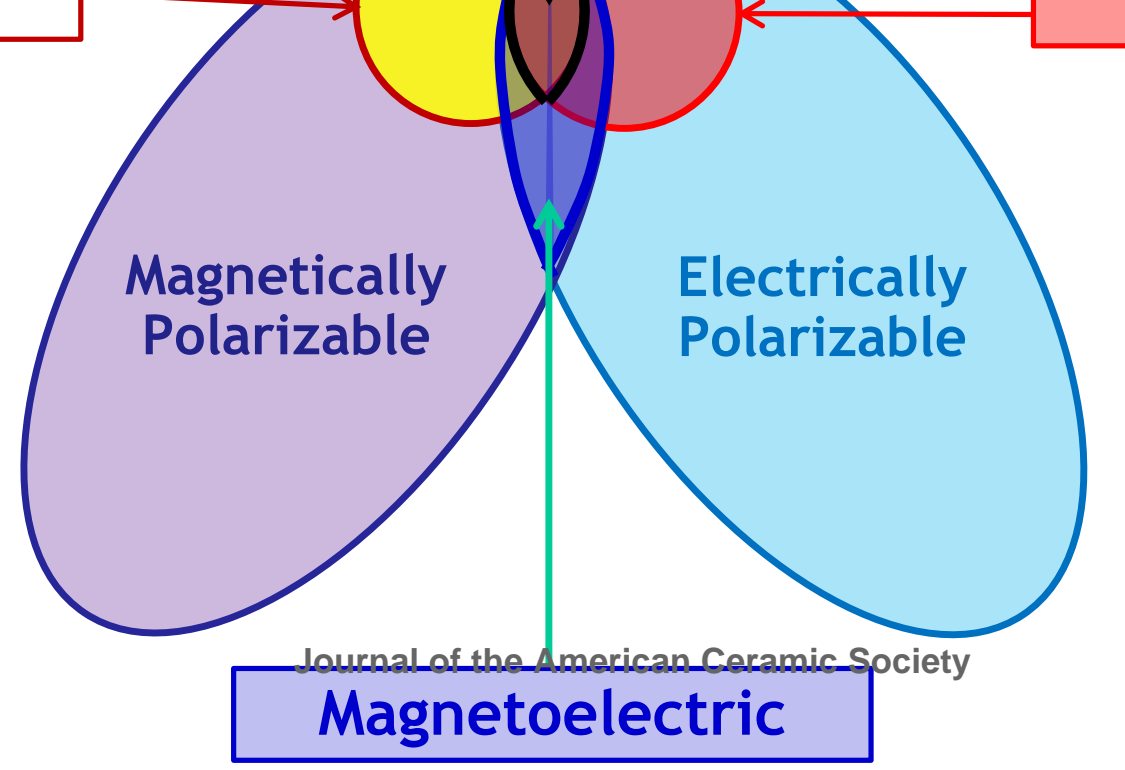




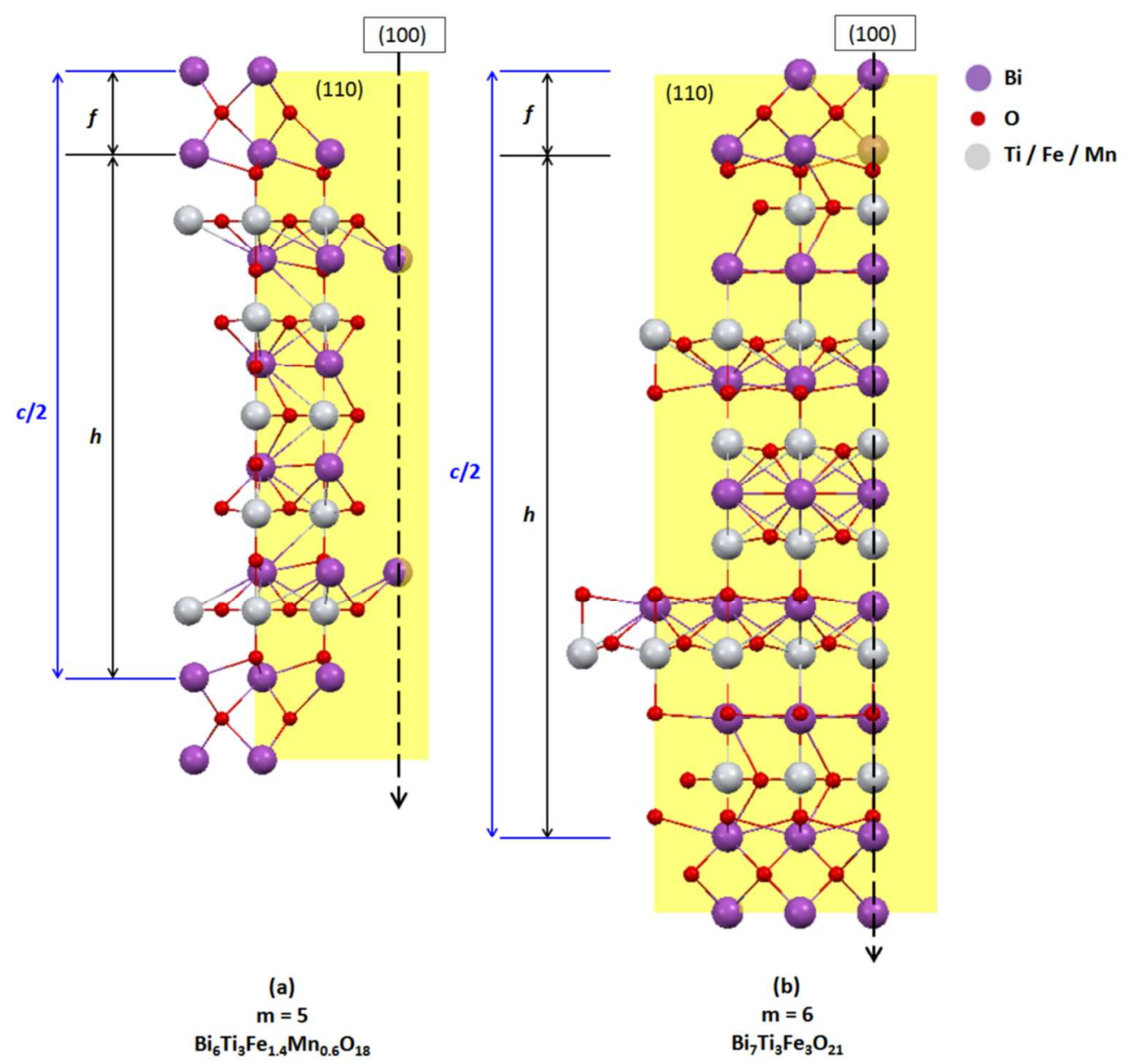

Fig. B.1. Projection (approximately down (101)) of half-unit cells of (a) $\mathrm{Bi}_{7} \mathrm{Ti}_{3} \mathrm{Fe}_{3} \mathrm{O}_{21}$ and (b) $\mathrm{Bi}_{6 \mathrm{Ti}} \mathrm{TF}_{1.6 \mathrm{Mn} 0.6018}$ displaying the in-plane lattice directions (100) (dashed arrow) and (110) (yellow plane). Drawn using Crystallographica v1.60d (Reference 62) and Mercury 3.0 Crystal Structure Visualization software (Reference 96$)$. $127 \times 118 \mathrm{~mm}(300 \times 300 \mathrm{DPI})$ 


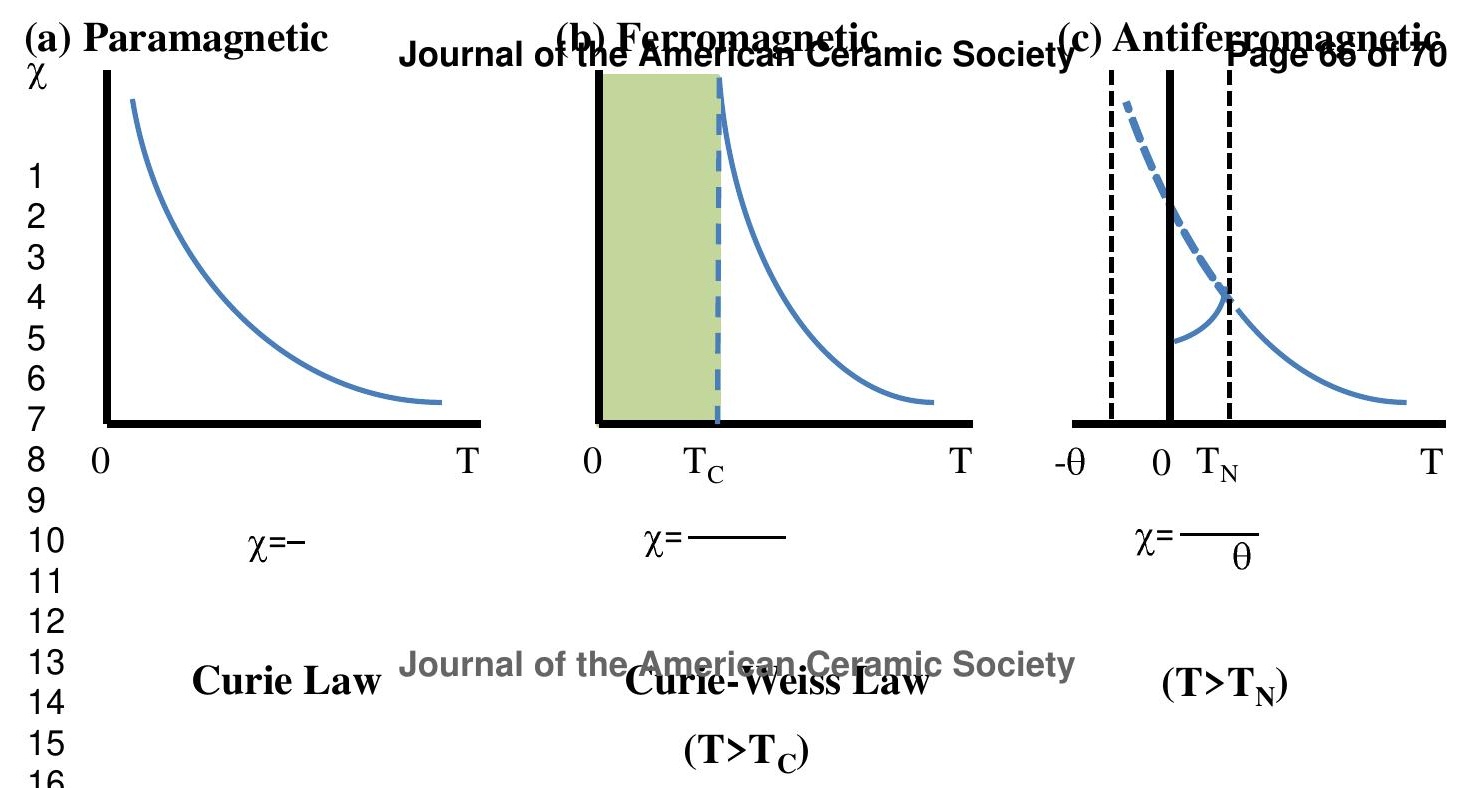




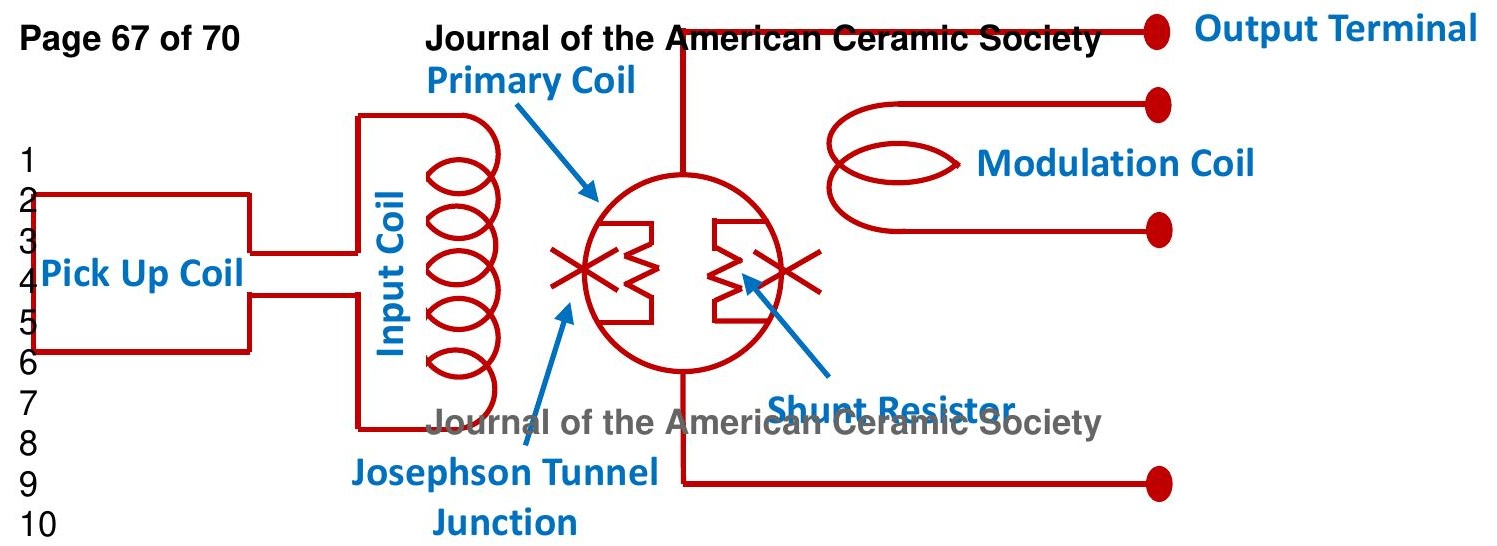


(a) Journal of the American Ceramiq Society

2

3

4

$5 \mathrm{M}$

6
FC Transition temperatures
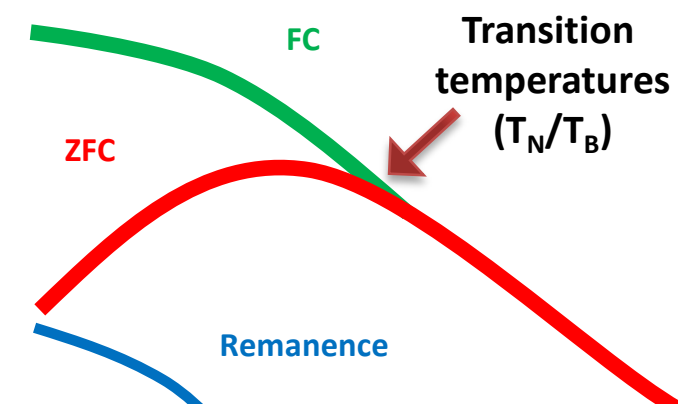

-
$\left(T_{N} / T_{B}\right)$

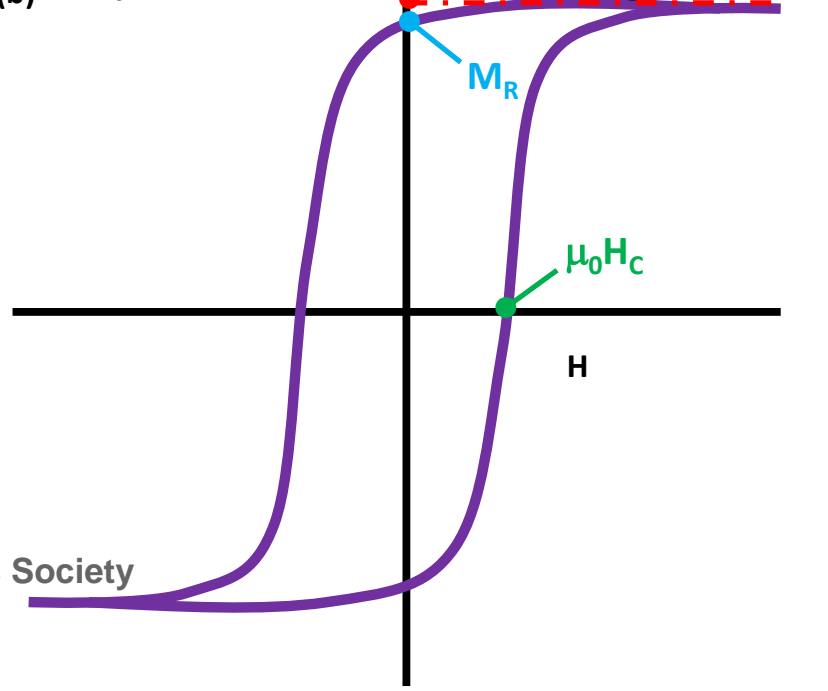


Table 1. Performed Volume Scans

\begin{tabular}{|c|c|c|c|c|c|}
\hline Method & $k$ & $\begin{array}{c}\text { Volume } V_{k} \\
{\left[\mu \mathrm{m}^{3}\right]}\end{array}$ & $\begin{array}{c}\text { smallest diameter } d_{k} \\
{[\mathrm{~nm}]}\end{array}$ & $\phi_{k}$ & $M_{r, k}$ \\
& & & & & \\
\hline Surface EDX & 1 & 2000 & $1000\left(\mathrm{~d}_{0}=5 \mu \mathrm{m}\right)$ & $0.010 \%$ & 2.1 \\
\hline Surface EDX & 2 & 450 & 350 & $0.006 \%$ & 1.1 \\
\hline Surface EDX & 3 & 120 & 100 & $0.005 \%$ & 1.0 \\
\hline Surface EDX & 4 & 28 & 60 & $0.001 \%$ & 0.2 \\
\hline Surface EDX & 5 & 7.2 & 20 & $0.008 \%$ & 1.7 \\
\hline TEM & 6 & 0.08 & 2 & $0.028 \%$ & 2.8 \\
\hline
\end{tabular}

